Advanced Nonlinear Studies 6 (2006), 333-357

\title{
Normalized Solutions to Strongly Indefinite Semilinear Equations
}

\author{
Boris Buffoni \\ School of Mathematics, École Polytechnique Fédérale-Lausanne, \\ SB/IACS/ANA Station 8, 1015 Lausanne, Switzerland \\ e-mail: boris.buffoni@epfl.ch \\ Maria J. Esteban, Eric Séré \\ CEREMADE (UMR CNRS 7534) Université de Paris-Dauphine, \\ Pl. du Maréchal de Lattre de Tassigny, 75775 Paris Cedex 16, France \\ esteban,sere@ceremade.dauphine.fr \\ Received 17 April 2006 \\ Communicated by Shair Ahmad
}

\begin{abstract}
In this paper we discuss the existence of normalized solutions of nonlinear elliptic PDEs in gaps of the essential spectrum of the corresponding differential operator. This issue can be of interest, for instance, in nonlinear optics or in crystalline models with impurities.
\end{abstract}

2000 Mathematics Subject Classification. 35J60, 35P30, 35J20, 35J10, 35Q55, 81Q05.

Key Words. Normalized solutions, nonlinear elliptic partial differential equations, Morse index, indefinite functionals, variational methods, penalization.

\section{Introduction}

Finding normalized solutions of strongly indefinite semilinear elliptic equations in unbounded domains has been a long standing question, the normalization being for instance in the space $L^{2}\left(\mathbb{R}^{d}\right)$. This kind of condition is natural for instance in quantum mechanics, where one looks for particles which have unit charge. In this paper we give an answer to this question for some particular nonlinearities, local and nonlocal. We study the two 
particular model equations

$$
-\Delta u(x)+p(x) u(x)=a(x)|u(x)|^{\gamma-1} u(x)+\lambda u(x), u \in H^{1}\left(\mathbb{R}^{d}\right), d \geq 1,
$$

and

$$
-\Delta u(x)+p(x) u(x)-\frac{\alpha}{|x|} u(x)+\beta\left(u^{2} * \frac{1}{|x|}\right) u=\lambda u(x), u \in H^{1}\left(\mathbb{R}^{d}\right), d \geq 3,
$$

together with the normalization condition

$$
\int_{\mathbb{R}^{d}} u^{2}(x) d x=1
$$

where $p \in L^{\infty}\left(\mathbb{R}^{d}\right)$ is periodic (and real valued), $\alpha, \beta>0, \gamma>1, a \in L^{\infty}\left(\mathbb{R}^{d}\right), a \geq 0$, $a \not \equiv 0$ and either $\lim _{|x| \rightarrow \infty} a(x)=0$ or $a \in L^{q}\left(\mathbb{R}^{d}\right)$ for some $q \in[1, \infty)$.

The (unknown) spectral parameter $\lambda$ is such that the unbounded self-adjoint linear operator in $L^{2}\left(\mathbb{R}^{d}\right) u \rightarrow-\Delta u+p u-\lambda u$ is invertible but not positive definite, that is, $\lambda$ is in a spectral gap of $u \rightarrow-\Delta u+p u$, denoted by $\left(\lambda_{0}-m, \lambda_{0}+l\right)$ with $m, l>0$ and $\lambda_{0} \in \mathbb{R}$.

In the case of Equation (1.1), we prove that under the above conditions, there exist $\lambda \in\left(\lambda_{0}-m, \lambda_{0}+l\right)$ and $u \in H^{1}\left(\mathbb{R}^{d}\right)$ such that

$$
-\Delta u(x)+p(x) u(x)=a(x)|u(x)|^{\gamma-1} u(x)+\lambda u(x) \text { in } \mathbb{R}^{d}, \quad \int_{\mathbb{R}^{d}} u^{2}(x) d x=1,
$$

if $1<\gamma<1+\frac{4}{d}(d \geq 3), 1<\gamma<3(d=1,2)$, if the function $a(x)$ goes slowly enough to 0 at $x$ approaches $\pm \infty$ and if, in addition, there is no solution of

$$
-\Delta u(x)+p(x) u(x)=a(x)|u(x)|^{\gamma-1} u(x)+\left(\lambda_{0}-m\right) u(x) \text { in } \mathbb{R}^{d},
$$

satisfying $0<\int_{\mathbb{R}^{d}} u^{2}(x) d x \leq 1$.

This last condition is difficult to verify, but this can be done. For instance, when $\|a\|_{L^{q\left(\mathbb{R}^{d}\right)}}$ is small enough either for $q=\infty$ or for some appropriate $q \in(1, \infty)$. For precise statements about Equation (1.1), see Theorems 1, 2 and 3 below. Let us just mention a particular case of Theorem 2 where this last condition can also be verified: if $d=1$, $1<\gamma<3, p$ is piecewise continuous and $a \geq 0$ is continuous with non-empty compact support, then there exist $\lambda \in\left(\lambda_{0}-m, \lambda_{0}+l\right)$ and $u \in H^{1}(\mathbb{R})$ such that

$$
-u^{\prime \prime}(x)+p(x) u(x)=a(x)|u(x)|^{\gamma-1} u(x)+\lambda u(x) \text { in } \mathbb{R}, \int_{\mathbb{R}} u^{2}(x) d x=1 .
$$

Note that the above results correspond to subcritical cases for Equation (1.1).

As far as Equation (1.2) is concerned, let us define $H_{ \pm} \subset H^{1}\left(\mathbb{R}^{d}\right)$ as the positive/negative spectral space associated with $-\Delta+p-\lambda_{0}$. Then, we prove the existence of a normalized solution for some $\lambda \in\left(\lambda_{0}, \lambda_{0}+m\right)$ if $0<\beta<\alpha$ and if 


$$
\begin{gathered}
\inf _{\substack{v \in H_{+} \\
\|v\|_{H^{1}}=1}} \int_{\mathbb{R}^{d}}|\nabla v|^{2}+\left(p-\lambda_{0}-\alpha|x|^{-1}\right) v^{2} d x>0, \\
\sup _{\substack{u \in H^{1}\left(\mathbb{R}^{d}\right) \\
\|u\| \|_{L^{2}} \leq 1}} \sup _{\substack{w \in H_{-} \\
\|w\|_{H^{1}}=1}} \int_{\mathbb{R}^{d}}|\nabla w|^{2}+\left(p-\lambda_{0}-\frac{\alpha}{|x|}\right) w^{2}+3 \beta\left(u^{2} * \frac{1}{|x|}\right) w^{2} d x<0 .
\end{gathered}
$$

Note that the above conditions are satisfied if $\alpha, \beta$ are small enough while still satisfying $0<\beta<\alpha$, thanks to the Hardy inequality : $\int_{\mathbb{R}^{d}}\left(f^{2} / x^{2}\right) d x \leq \frac{4}{(d-2)^{2}} \int_{\mathbb{R}^{d}}|\nabla f|^{2} d x$, for all $f \in H^{1}\left(\mathbb{R}^{d}\right)$ if $d \geq 3$. From the point of view of physics, the relevant dimension for Equation (1.2) is $d=3$. The function $p(x)$ can be interpreted as the Coulomb potential in a three-dimensional periodic crystal. Now, in this crystal, assume that the atomic nucleus located at the origin of coordinates has been replaced by a nucleus of higher atomic number (commonly called an "impurity", see [8]). This impurity generates the additional Coulomb potential $-\alpha|x|^{-1}$. In some situations it can bind two electrons. To study this phenomenon, we make the so-called "restricted Hartree-Fock approximation" (see e.g. [15]), in which the two electrons have the same spatial wave function $u$, one electron has spin "up", the other has spin "down". This gives (1.2). In this interpretation, the nonlinear term $\beta\left(u^{2} * \frac{1}{|x|}\right)$ is the repulsive potential generated by an electron and felt by the other. The eigenvalue $\lambda$ is the energy of each electron.

Our approach to prove the main results of this paper is the one developed in [7] for the Dirac-Fock equations and is closely related to the theory of bifurcation from the essential spectrum, particularly [14]. Note that of course much more general nonlinear elliptic equations can be treated by the same arguments used to deal with (1.1) and (1.2). In Section 3 we give an abstract version of the theorems showing the requirements in the general case.

\section{General setting}

We are interested in problems of the form

$$
-\Delta u(x)+p(x) u(x)+V(x) u(x)=\tilde{N}(u)(x)+\lambda u(x) \text { in } \mathbb{R}^{d}, \quad \int_{\mathbb{R}^{d}} u^{2}(x) d x=1,
$$

where $\lambda$ is an unknown "spectral" parameter, $p \in L^{\infty}\left(\mathbb{R}^{d}\right)$ is periodic and $\tilde{N}(u)$ is a nonlinear function (or functional) of $u$, which is the derivative of a real-valued functional $\phi$. Indeed, for Equation (1.1), $\tilde{N}(u)=a(x)|u|^{\gamma-1} u$ and $\phi(u)=\frac{1}{\gamma+1} \int_{\mathbb{R}^{d}} a|u|^{\gamma+1}$, while for $(1.2), \tilde{N}(u)=\frac{\alpha}{|x|} u-\beta\left(|u|^{2} * \frac{1}{|x|}\right) u$ and $\phi(u)=\frac{\alpha}{2} \int_{\mathbb{R}^{d}} \frac{u^{2}}{|x|}-\frac{\beta}{4} \iint_{\mathbb{R}^{d} \times \mathbb{R}^{d}} \frac{u^{2}(x) u^{2}(y)}{|x-y|}$. Being a solution of $(2.6)$ is understood in the weak sense:

$$
\int_{\mathbb{R}^{d}}\{\nabla u(x) \cdot \nabla v(x)+p(x) u(x) v(x)-\lambda u(x) v(x)\} d x=\int_{\mathbb{R}^{d}} \tilde{N}(u)(x) v(x) d x
$$

for all $v \in H^{1}\left(\mathbb{R}^{d}\right)$. The unknown spectral parameter $\lambda$ is such that the unbounded selfadjoint linear operator in $L^{2}\left(\mathbb{R}^{d}\right) u \rightarrow-\Delta u+p u-\lambda u$ is invertible but not positive definite, 
that is, $\lambda$ is in a spectral gap of $u \rightarrow-\Delta+p u$. That such a spectral gap may exist when $p$ is not constant is a well-known phenomenon (see e.g. $[13,14,16])$. We denote it by $\left(\lambda_{0}-m, \lambda_{0}+l\right)$ with $m, l>0$ and $\lambda_{0} \in \mathbb{R}$ :

$\lambda_{0}-m \in \sigma(-\Delta u+p u), \lambda_{0}+l \in \sigma(-\Delta u+p u)$ and $\left(\lambda_{0}-m, \lambda_{0}+l\right) \cap \sigma(-\Delta u+p u)=\emptyset$.

For $u \in H^{1}\left(\mathbb{R}^{d}\right)$, the functional $v \rightarrow \int_{\mathbb{R}^{d}}\{-\Delta u+p u-\lambda u\} v d x$ is well defined if $v \in H^{1}\left(\mathbb{R}^{d}\right)$ and thus $-\Delta u+p u-\lambda u \in H^{-1}\left(\mathbb{R}^{d}\right)$. If we now introduce in $H^{1}\left(\mathbb{R}^{d}\right)$ the inner product

$$
\langle u, v\rangle_{H^{1}}:=\int_{\mathbb{R}^{d}} \nabla u \cdot \nabla v+\left(p-\inf _{\mathbb{R}^{d}} p+1\right) u v d x,
$$

we can identify $H^{1}\left(\mathbb{R}^{d}\right)$ and its dual $H^{-1}\left(\mathbb{R}^{d}\right)$ and also $-\Delta u+p u$ can be identified with $L u=(-\Delta+p-\inf p+1)^{-1}(-\Delta u+p u) \in H^{1}\left(\mathbb{R}^{d}\right)$. This defines a bounded operator $L$ on $H^{1}\left(\mathbb{R}^{d}\right)$. In other words,

$$
\int_{\mathbb{R}^{d}}\{-\Delta u+p u\} v d x=\langle L u, v\rangle_{H^{1}} \text { for all } v \in H^{1}\left(\mathbb{R}^{d}\right) .
$$

In the same way, there is a nonlinear operator $N: H^{1}\left(\mathbb{R}^{d}\right) \rightarrow H^{1}\left(\mathbb{R}^{d}\right)$ such that

$$
\int_{\mathbb{R}^{d}} \tilde{N}(u) v d x=\langle N(u), v\rangle_{H^{1}} \text { for all } v \in H^{1}\left(\mathbb{R}^{d}\right),
$$

and a linear operator $A: H^{1}\left(\mathbb{R}^{d}\right) \rightarrow H^{1}\left(\mathbb{R}^{d}\right)$ such that

$$
\int_{\mathbb{R}^{d}} u v d x=\langle A u, v\rangle_{H^{1}} \text { for all } v \in H^{1}\left(\mathbb{R}^{d}\right) .
$$

With these notations, the problem is to find $u \in H^{1}\left(\mathbb{R}^{d}\right)$ such that

$$
L u=N(u)+\lambda A u,\langle A u, u\rangle_{H^{1}\left(\mathbb{R}^{d}\right)}=1 .
$$

Note that $L$ is bounded, self-adjoint and invertible, but neither positive nor negative definite, whereas $A$ is bounded, self-adjoint and positive definite, but has no bounded inverse. Moreover $L A=A L$ and $N(u)$ is the gradient, for the $H^{1}$-scalar product, of the $C^{2}$ functional $\phi(u)$ defined by

$$
\phi(u):=\frac{1}{1+\gamma} \int_{\mathbb{R}^{d}} a(x)|u|^{\gamma+1} d x \quad \text { in the case of Equation (1.1) },
$$

$\phi(u):=\frac{\alpha}{2} \int_{\mathbb{R}^{d}} \frac{|u|^{2}}{|x|} d x-\frac{\beta}{4} \iint_{\mathbb{R}^{d} \times \mathbb{R}^{d}} \frac{u^{2}(x) u^{2}(y)}{|x-y|} d x d y \quad$ in the case of Equation (1.2).

The main advantages of these notations are that they are closely related to the variational structure of the problem and that all linear operators are bounded. They lead to a general and abstract framework, but first let us state our main results for $(2.6)$, which ensures the existence of a normalized solution for at least one $\lambda \in\left(\lambda_{0}-m, \lambda_{0}+l\right)$.

Let us start with the first case $\tilde{N}(u)=a(x)|u|^{\gamma-1} u$. 
Theorem 1. Let $d \geq 2$, let $p \in L^{\infty}\left(\mathbb{R}^{d}\right)$ be periodic, let $a \in L^{\infty}\left(\mathbb{R}^{d}\right), a \geq 0, a \neq \equiv$, let $\gamma$ satisfy $1<\gamma<1+\frac{4}{d}$ and define

$$
\tau(\gamma)=2-\frac{d}{2}(\gamma-1)>0
$$

Assume that

$$
\text { either } a(x) \rightarrow 0 \text { as }|x| \rightarrow+\infty \text { or } a \in L^{q}\left(\mathbb{R}^{d}\right) \text { for some } q \in[1,+\infty)
$$

and for all large $|x|$,

$$
a(x) \geq C|x|^{-\tau} \text { for some } \tau<\tau(\gamma) \text { and some constant } C>0 .
$$

Then either there exist $\lambda \in\left(\lambda_{0}-m, \lambda_{0}+l\right)$ and $u \in H^{1}\left(\mathbb{R}^{d}\right)$ such that

$$
-\Delta u(x)+p(x) u(x)=a(x)|u(x)|^{\gamma-1} u(x)+\lambda u(x) \text { in } \mathbb{R}^{d}, \int_{\mathbb{R}^{d}} u^{2}(x) d x=1,
$$

or there is a solution of

$$
-\Delta u(x)+p(x) u(x)=a(x)|u(x)|^{\gamma-1} u(x)+\left(\lambda_{0}-m\right) u(x) \text { in } \mathbb{R}^{d},
$$

satisfying $0<\int_{\mathbb{R}^{d}} u^{2}(x) d x \leq 1$,

(or both hold simultaneously).

In the unidimensional case we can prove the impossibility of the second case in the above alternative (note that the hypothesis (2.9) on the decay of $a(x)$ at $\pm \infty$ is no longer needed):

Theorem 2. Let $p \in L^{\infty}(\mathbb{R})$ be periodic and piecewise continuous, let $a \geq 0$, $a \not \equiv 0$, be continuous and let $\gamma$ satisfy $1<\gamma<3$. Assume that $a \in L^{\infty}(\mathbb{R})$ and that either $a(x) \rightarrow 0$ as $|x| \rightarrow+\infty$ or $a \in L^{q}(\mathbb{R})$ for some $q \in[1,+\infty)$. Assume furthermore that $\int_{\mathbb{R}}\{|x| a(x)\}^{2 /(3-\gamma)} d x<\infty$. Then there exist $\lambda \in\left(\lambda_{0}-m, \lambda_{0}+l\right)$ and $u \in H^{1}(\mathbb{R})$ such that

$$
-u^{\prime \prime}(x)+p(x) u(x)=a(x)|u(x)|^{\gamma-1} u(x)+\lambda u(x) \text { in } \mathbb{R}, \quad \int_{\mathbb{R}} u^{2}(x) d x=1 .
$$

In the higher dimensional case, a similar result can also be proved if the function $a$ is "small enough":

Theorem 3. Under the assumptions of Theorem 1, if $\|a\|_{L^{q}\left(\mathbb{R}^{d}\right)}$ is small enough, either for $q=\infty$ or for some $q>\frac{2}{1+\frac{4}{d}-\gamma}$, then there exist $\lambda \in\left(\lambda_{0}-m, \lambda_{0}+l\right)$ and $u \in H^{1}\left(\mathbb{R}^{d}\right)$ such that

$$
-\Delta u(x)+p(x) u(x)=a(x)|u(x)|^{\gamma-1} u(x)+\lambda u(x) \text { in } \mathbb{R}^{d}, \quad \int_{\mathbb{R}^{d}} u^{2}(x) d x=1 .
$$


In the case of Equation (1.2), when $\tilde{N}(u)=\frac{\alpha}{|x|} u-\beta\left(|u|^{2} * \frac{1}{|x|}\right) u$, we can prove the following.

Theorem 4. If $p \in L^{\infty}\left(\mathbb{R}^{d}\right)$ is periodic, $d \geq 3,0<\beta<\alpha$ and if (1.5) holds, then there exist $\lambda \in\left(\lambda_{0}, \lambda_{0}+l\right)$ and $u \in H^{1}\left(\mathbb{R}^{d}\right)$ such that

$$
-\Delta u(x)+p(x) u(x)-\frac{\alpha}{|x|} u(x)+\beta\left(u^{2} * \frac{1}{|x|}\right) u=\lambda u(x) \text { in } \mathbb{R}^{d}, \quad \int_{\mathbb{R}^{d}} u^{2}(x) d x=1 .
$$

The main new feature of the above theorems is that these are not results "in the small" of the type "bifurcation from the essential spectrum", although many technical steps are directly inspired by previous works in this field, particularly by [14] and [3, 10, 11, 12]. Our approach is the one developed in [7] for the Dirac-Fock equations based on an unconstrained penalization, a variational Lyapunov-Schmidt reduction $[1,5,6,4]$ and the mountain-pass theorem (see e.g. [9]). In the superlinear case (Equation (1.1)) some compactness is assumed in this paper (namely, we assume that at least in a weak sense $a$ is small at infinity). In the case of Equation (1.2) the uniform spectral condition (1.5) is enough to ensure the necessary compactness.

\section{Abstract results.}

We consider instead of $H^{1}\left(\mathbb{R}^{d}\right)$ a general real Hilbert space $H$ with inner-product $\langle\cdot, \cdot\rangle$ and norm $\|\cdot\|$, and the equation

$$
\left\{\begin{array}{l}
L u=N(u)+\lambda A u \text { in } H \\
\langle A u, u\rangle=1
\end{array}\right.
$$

where $L: H \rightarrow H$ is a bounded linear self-adjoint operator with bounded inverse (this means that, in the examples, we assume $\lambda_{0}=0$, without loss of generality). The bounded linear operator $A: H \rightarrow H$ is self-adjoint, positive definite, but $0 \in \sigma(A)$ is allowed. Moreover $L A=A L$.

Let $H_{+}$and $H_{-}$be the eigenspaces corresponding to $\sigma(L) \cap \mathbb{R}_{+}$and $\sigma(L) \cap \mathbb{R}_{-}$, supposed nontrivial; they satisfy

$$
\langle L u, u\rangle \geq \delta\|u\|^{2} \forall u \in H_{+} \text {and }\langle L u, u\rangle \leq-\delta\|u\|^{2} \forall u \in H_{-}
$$

for some $\delta>0$. We denote by $P$ and $I-P$ the orthogonal projections on $H_{+}$and $H_{-}$. Let

$$
l=\inf _{u \in H_{+} \backslash\{0\}} \frac{\langle L u, u\rangle}{\langle A u, u\rangle} \geq \inf _{u \in H_{+} \backslash\{0\}} \frac{\delta\|u\|^{2}}{\langle A u, u\rangle} \geq \frac{\delta}{\|A\|}>0
$$

and

$$
m=\inf _{u \in H_{-} \backslash\{0\}} \frac{-\langle L u, u\rangle}{\langle A u, u\rangle} \geq \inf _{u \in H_{-} \backslash\{0\}} \frac{\delta\|u\|^{2}}{\langle A u, u\rangle} \geq \frac{\delta}{\|A\|}>0 .
$$

Let $\phi \in C^{2}(H, \mathbb{R})$ be such that

(H1) $N=\nabla \phi$ on $H$ with $\phi(0)=0$ and $\phi^{\prime}(0)=N(0)=0$. 
Together with $A$, we also consider $A_{\mu}$ defined by

$$
A_{\mu} u=A u+\mu L P u-\mu L(I-P) u \text { and } \mu>0 .
$$

Our variational method is based on the functional

$$
J_{r, \mu}(u)=\frac{1}{2}\langle L u, u\rangle-\phi(u)-\psi_{r, \mu}(u), \quad u \in U_{\mu}
$$

where $U_{\mu}=\left\{u \in H:\left\langle A_{\mu} u, u\right\rangle<1\right\}, \psi_{r, \mu}(u)$ is a penalization term defined by

$$
\psi_{r, \mu}(u)=f_{r}\left(\left\langle A_{\mu} u, u\right\rangle\right) \text { with } f_{r}(s)=\frac{s^{r}}{1-s}(0 \leq s<1),
$$

and $r>2$ is a parameter that will be chosen large (in fact we shall let $r \rightarrow \infty$ ).

Here are some properties of the penalization $\psi_{r, \mu}$ for $u \in U_{\mu}$ and $v, v_{1}, v_{2} \in H$ :

$$
\left\langle\nabla \psi_{r, \mu}(u), v\right\rangle=2 f_{r}^{\prime}\left(\left\langle A_{\mu} u, u\right\rangle\right)\left\langle A_{\mu} u, v\right\rangle
$$

with

$$
f_{r}^{\prime}(s)=\frac{r s^{r-1}}{1-s}+\frac{s^{r}}{(1-s)^{2}}>\frac{r}{s} f_{r}(s)>0 \quad(0<s<1)
$$

and

$$
\left\langle\psi_{r, \mu}^{\prime \prime}(u) v_{1}, v_{2}\right\rangle=2 f_{r}^{\prime}\left(\left\langle A_{\mu} u, u\right\rangle\right)\left\langle A_{\mu} v_{1}, v_{2}\right\rangle+4 f_{r}^{\prime \prime}\left(\left\langle A_{\mu} u, u\right\rangle\right)\left\langle A_{\mu} u, v_{1}\right\rangle\left\langle A_{\mu} u, v_{2}\right\rangle
$$

with

$$
f_{r}^{\prime \prime}(s)=\frac{r(r-1) s^{r-2}}{1-s}+\frac{2 r s^{r-1}}{(1-s)^{2}}+\frac{2 s^{r}}{(1-s)^{3}}>0(0<s<1) .
$$

In particular $\psi_{r, \mu}$ is convex and, for all $u \in U_{\mu},\left\langle\nabla \psi_{r, \mu}(u), u\right\rangle \geq r \psi_{r, \mu}(u)$.

Next we assume

(C1) For every $v \in H_{+}$such that $\langle A v, v\rangle<1$, the map

$$
H_{-} \ni w \mapsto \frac{1}{2}\langle L(v+w), v+w\rangle-\phi(v+w)
$$

has negative definite Hessian at every $w \in H_{-}$such that $\langle A(v+w), v+w\rangle<1$.

Note that $w \mapsto-J_{r, \mu}(v+w)$ is coercive on $\left\{w \in H_{-}, v+w \in U_{\mu}\right\}$. We now perform a variational Lyapunov-Schmidt reduction in the spirit of the one in $[1,6,5]$. From $(\mathrm{C} 1)$ and the convexity of the function $\psi_{r, \mu}$, we see that for all $v \in H_{+} \cap U_{\mu}$, there exists a unique $w=: g_{r, \mu}(v) \in H_{-}$such that $\left\langle A_{\mu}\left(v+g_{r, \mu}(v)\right), v+g_{r, \mu}(v)\right\rangle<1$,

$$
J_{r, \mu}\left(v+g_{r, \mu}(v)\right)=\max \left\{J_{r, \mu}(v+w): w \in H_{-},\left\langle A_{\mu}(v+w), v+w\right\rangle<1\right\}
$$

and

$$
(I-P) \nabla J_{r, \mu}\left(v+g_{r, \mu}(v)\right)=0
$$


which is equivalent to

$$
\begin{aligned}
0=L g_{r, \mu}(v)-(I-P) N( & \left.+g_{r, \mu}(v)\right) \\
& -2 f_{r}^{\prime}\left(\left\langle A_{\mu}\left(v+g_{r, \mu}(v)\right), v+g_{r, \mu}(v)\right\rangle\right) A g_{r, \mu}(v) .
\end{aligned}
$$

By the Implicit Function Theorem, $g_{r, \mu}$ is of class $C^{1}$ and $g_{r, \mu}(0)=0$, because the above equality holds true when $v=0$ and $g_{r, \mu}(v)=0$.

For all $r>2$ we can now define a reduced functional $F_{r, \mu}: H_{+} \cap U_{\mu} \rightarrow \mathbb{R}$ by

$$
\begin{aligned}
F_{r, \mu}(v)=J_{r, \mu}\left(v+g_{r, \mu}(v)\right) & \\
=\frac{1}{2}\langle L v, v\rangle+\frac{1}{2}\left\langle L g_{r, \mu}(v), g_{r, \mu}(v)\right\rangle-\phi\left(v+g_{r, \mu}(v)\right) & \quad-f_{r}\left(\left\langle A_{\mu}\left(v+g_{r, \mu}(v)\right), v+g_{r, \mu}(v)\right\rangle\right),
\end{aligned}
$$

which is of class $C^{1}$ and satisfies

$$
\nabla_{H_{+}} F_{r, \mu}(v) \stackrel{(3.14)}{=} P \nabla_{H} J_{r, \mu}\left(v+g_{r, \mu}(v)\right)
$$

This shows that $F_{r, \mu}$ is in fact of class $C^{2}$ and

$$
\begin{aligned}
& F_{r, \mu}^{\prime \prime}(v)\left(v_{1}, v_{2}\right)=J_{r, \mu}^{\prime \prime}\left(v+g_{r, \mu}(v)\right)\left(v_{1}, v_{2}+g_{r, \mu}^{\prime}(v) v_{2}\right) \\
& \quad \stackrel{(3.14)}{=} J_{r, \mu}^{\prime \prime}\left(v+g_{r, \mu}(v)\right)\left(v_{1}+g_{r, \mu}^{\prime}(v) v_{1}, v_{2}+g_{r, \mu}^{\prime}(v) v_{2}\right), \quad v_{1}, v_{2} \in H_{+} .
\end{aligned}
$$

Also, by (3.15),

$$
\begin{aligned}
L\left(g_{r, \mu}^{\prime}(0) v\right)=(I-P) N^{\prime}(0)\left(v+g_{r, \mu}^{\prime}(0) v\right)+2 f_{r}^{\prime}(0) & A_{\mu}\left(g_{r, \mu}^{\prime}(0) v\right) \\
= & (I-P) N^{\prime}(0)\left(v+g_{r, \mu}^{\prime}(0) v\right),
\end{aligned}
$$

which shows that

$$
g_{r, \mu}^{\prime}(0) v=\left\{(I-P)\left(L-N^{\prime}(0)\right)\right\}^{-1}(I-P) N^{\prime}(0) v
$$

does not depend on $r, \mu$ and $A$, and neither does

$$
F_{r, \mu}^{\prime \prime}(0)=\left(P+g_{r, \mu}^{\prime}(0)\right)^{T}\left(L-\phi^{\prime \prime}(0)\right)\left(P+g_{r, \mu}^{\prime}(0)\right) .
$$

Indeed they only depend on $L$ and $N^{\prime}(0)$. As a consequence, the fact that

$$
\inf \left\{\left\langle F_{r, \mu}^{\prime \prime}(0) v, v\right\rangle \mid v \in H_{+},\|v\|=1\right\}>0
$$

holds true does not depend on $r$ and $\mu$, and indeed depends only on $L$ and $N^{\prime}(0)$. Hence (3.18) holds for $L$ and $\phi$ if and only if it does for $L$ and for the function $H \ni u \rightarrow$ $\frac{1}{2} \phi^{\prime \prime}(0)(u, u)$ (defined on $H$, without corresponding $A_{\mu}$ ). Thus, we get that

$$
\left\langle F_{r, \mu}^{\prime \prime}(0) v, v\right\rangle \geq\left\langle\left(L-\phi^{\prime \prime}(0)\right) v, v\right\rangle
$$


and that (3.18) holds if $\inf \left\{\left\langle\left(L-\phi^{\prime \prime}(0)\right) v, v\right\rangle \mid v \in H_{+},\|v\|=1\right\}>0$. Hence, we further assume

$$
\inf \left\{\left\langle\left(L-\phi^{\prime \prime}(0)\right) v, v\right\rangle \mid v \in H_{+},\|v\|=1\right\}>0 .
$$

Clearly, for all $r>2, \mu>0$ and $v \in H_{+}, \lim _{t \rightarrow 1^{-}} F_{r, \mu}(t v)=-\infty$ if $\left\langle A_{\mu} v, v\right\rangle=1$.

As a consequence, $F_{r, \mu}$ has a mountain-pass structure:

$c_{r, \mu}:=\inf \left\{\max _{t \in[0,1]} F_{r, \mu}(\gamma(t)): \gamma \in C\left([0,1], U_{\mu} \cap H_{+}\right), \gamma(0)=0, F_{r, \mu}(\gamma(1))<0\right\}>0$.

This mountain-pass structure allows us to find a Palais-Smale sequence $\left\{v_{n}\right\}_{n}$ satisfying

$$
F_{r, \mu}\left(v_{n}\right) \rightarrow c_{r, \mu}, \quad \nabla F_{r, \mu}\left(v_{n}\right) \rightarrow 0,
$$

which we call a mountain-pass critical sequence. If we can prove some compactness for the sequence $\left\{v_{n}\right\}_{n}$, we will be able to pass to the limit along some subsequence and find $v$ such that $u=v+g_{r, \mu}(v)$ is a solution to

$$
L(u)=N(u)+\lambda_{r, \mu} A_{\mu} u,
$$

with $\lambda_{r, \mu}=2 f_{r}^{\prime}\left(\left\langle A_{\mu} u, u\right\rangle\right)$.

The functional $F_{r, \mu}$ is defined on the bounded set

$$
\left\{v \in H_{+}:\langle A v, v\rangle+\mu\langle L v, v\rangle<1\right\} \stackrel{(3.11)}{\complement}\left\{v \in H_{+}:\|v\|<(\mu \delta)^{-1 / 2}\right\} .
$$

For an analogous reason, $J_{r, \mu}$ too is defined on a bounded domain (namely $U_{\mu}$ ). If $r_{1} \leq r_{2}$ and $\mu_{1} \leq \mu_{2}$, we get $c_{r_{1}, \mu_{2}} \leq c_{r_{2}, \mu_{1}}$ because $J_{r_{1}, \mu_{2}} \leq J_{r_{2}, \mu_{1}}$ on $U_{\mu_{2}}$.

Define $c_{\infty}:=\sup _{r>2, \mu>0} c_{r, \mu}$. The main abstract theorem in this paper states the following.

Theorem 5. In addition to hypotheses (H1), (C1) and (C2), we assume that there is a constant $l^{\prime}<l$ such that :

(i) for $r$ large enough and $\mu>0$ small enough, there exists a mountain-pass critical sequence $\left(v_{n}\right)$ of $F_{r, \mu}$ at level $c_{r, \mu}$ satisfying

$$
\limsup _{n \rightarrow+\infty} f_{r}^{\prime}\left(\left\langle A_{\mu} u_{n}, u_{n}\right\rangle\right) \leq l^{\prime} / 2, \text { where } u_{n}=v_{n}+g_{r, \mu}\left(v_{n}\right) \text {, }
$$

and such that the sequence $\left(u_{n}\right)$ is relatively compact in $H$.

(ii) Any sequence $\left(\bar{u}_{k}\right)$ of the form $\bar{u}_{k}=\bar{v}_{k}+g_{r_{k}, \mu_{k}}\left(\bar{v}_{k}\right)$ with $\bar{v}_{k}$ critical point of $F_{r_{k}, \mu_{k}}$ at level $c_{r_{k}, \mu_{k}}\left(r_{k} \rightarrow \infty, \mu_{k} \rightarrow 0\right)$, satisfying

$$
\limsup _{k \rightarrow+\infty} f_{r_{k}}^{\prime}\left(\left\langle A_{\mu_{k}} \bar{u}_{k}, \bar{u}_{k}\right\rangle\right) \leq l^{\prime} / 2
$$

is relatively compact in $H$.

Under these assumptions, we have $c_{\infty}<\infty$ and the following alternative: 
1. either there exists $\bar{u} \in H \backslash\{0\}$ and $\bar{\lambda} \in[0, l)$ such that

$$
L \bar{u}-N(\bar{u})=\bar{\lambda} A \bar{u}, \quad\langle A \bar{u}, \bar{u}\rangle=1 \quad \text { and } \quad 0<\frac{1}{2}\langle L \bar{u}, \bar{u}\rangle-\phi(\bar{u})=c_{\infty},
$$

2. or there exists $\bar{u} \in H \backslash\{0\}$ such that

$$
L \bar{u}-N(\bar{u})=0, \quad\langle A \bar{u}, \bar{u}\rangle<1 \quad \text { and } 0<\frac{1}{2}\langle L \bar{u}, \bar{u}\rangle-\phi(\bar{u})=c_{\infty},
$$

(or both).

Proof of Theorem 5. Step 1. $r<+\infty$ large, $\mu>0$ small.

We consider a sequence $\left(v_{n}\right)$ in the domain of $F_{r, \mu}$, relatively compact in $H$, satisfying (3.20) and such that $\nabla F_{r, \mu}\left(v_{n}\right) \rightarrow 0$ in $H_{+}$and $\lim _{n \rightarrow \infty} F_{r, \mu}\left(v_{n}\right)=c_{r, \mu}$. Clearly this sequence remains away from the boundary of the domain of $F_{r, \mu}$. We shall use the notation

$$
w_{n}=g_{r, \mu}\left(v_{n}\right), u_{n}=v_{n}+w_{n}, \text { and } \lambda_{n}=2 f_{r}^{\prime}\left(\left\langle A_{\mu} u_{n}, u_{n}\right\rangle\right),
$$

where $\left\langle A_{\mu} u_{n}, u_{n}\right\rangle<1$. By assumption (3.20), if $r$ is large enough and $\mu$ small enough, we may assume $\lambda_{n} \rightarrow \lambda_{r, \mu} \in\left[0, l^{\prime}\right] \subset[0, l)$.

We can apply the relative compactness assumption to infer the existence of a subsequence of $\left(v_{n}\right)$, denoted still by $\left(v_{n}\right)$, which converges towards some $v_{r, \mu} \in H_{+}$. If we define $u_{r, \mu}=v_{r, \mu}+g_{r, \mu}\left(v_{r, \mu}\right)$, by continuity we know that

$$
\begin{gathered}
\nabla F_{r, \mu}\left(v_{r, \mu}\right)=0, \quad F_{r, \mu}\left(v_{r, \mu}\right)=c_{r, \mu},\left\langle A_{\mu} u_{r, \mu}, u_{r, \mu}\right\rangle<1, \\
\lambda_{r, \mu}=2 f_{r}^{\prime}\left(\left\langle A_{\mu} u_{r, \mu}, u_{r, \mu}\right\rangle\right) \in\left[0, l^{\prime}\right] \subset[0, l),
\end{gathered}
$$

and

$$
0<\frac{1}{2}\left\langle L u_{r, \mu}, u_{r, \mu}\right\rangle-\phi\left(u_{r, \mu}\right)=c_{r, \mu}+\psi_{r}\left(u_{r, \mu}\right)<c_{r, \mu}+(2 r)^{-1} \lambda_{r, \mu} .
$$

Step 2. $r_{n} \rightarrow+\infty, \mu_{n} \rightarrow 0$.

We can use Step 1 to obtain a sequence of critical points for $F_{r_{n}, \mu_{n}}$ satisfying (3.22), (3.23), (3.24) and such that $\left(r_{n}\right)$ and $\left(\mu_{n}\right)$ are monotone sequences going to $\infty$ and 0 .

We use (3.21) to extract a subsequence, still denoted by $\left(\bar{u}_{r_{n}, \mu_{n}}\right)$, converging to some $\bar{u} \in H$, which satisfies,

$$
\begin{gathered}
L \bar{u}=N(\bar{u})+\bar{\lambda} A \bar{u}, \quad\langle A \bar{u}, \bar{u}\rangle \leq 1, \quad 0 \leq \bar{\lambda} \leq l^{\prime}<l, \\
0<c_{r_{1}, \mu_{1}} \leq \frac{1}{2}\langle L \bar{u}, \bar{u}\rangle-\phi(\bar{u})=c_{\infty} .
\end{gathered}
$$

Now, either $\langle A \bar{u}, \bar{u}\rangle=1$ or $\langle A \bar{u}, \bar{u}\rangle<1$. When the latter happens,

$$
0 \leq \bar{\lambda}=\lim _{n \rightarrow \infty} f_{r_{n}}^{\prime}\left(\left\langle A \bar{u}_{r_{n}}, \bar{u}_{r_{n}}\right\rangle\right) \leq \limsup _{n \rightarrow \infty} f_{r_{n}}^{\prime}\left(\frac{1+\langle A \bar{u}, \bar{u}\rangle}{2}\right)=0,
$$

that is, $\bar{\lambda}=0$. This leads to the alternative of the statement. 
Corollary 6. Assume that, for every $s \in(-m, l)$, conditions (C1), (C2) and the assumptions of Theorem 5 remain true if $L$ is replaced everywhere by $L-s A$ and $l$ by $l-s$, and $F_{r, \mu}, c_{r, \mu}$ and $g_{r, \mu}$ modified accordingly. Then

1. either there exists $u \in H \backslash\{0\}$ and $\lambda \in(-m, l)$ such that

$$
L u-N(u)=\lambda A u, \quad\langle A u, u\rangle=1,
$$

2. or, for all $\lambda \in(-m, l)$, there exists $u_{\lambda} \in H \backslash\{0\}$ such that

$$
L u_{\lambda}-N\left(u_{\lambda}\right)=\lambda A u_{\lambda}, \quad\left\langle A u_{\lambda}, u_{\lambda}\right\rangle<1 .
$$

Proof. Apply Theorem 5 to the operator $L-s A$ for any $s \in(-m, l)$ instead of $L$.

\section{Proofs of Theorems 1, 2 and 3.}

In this section we consider a class of problems containing (1.1) as a particular case. We will deal with the general problem (3.10) and make the following hypotheses on $N$. First, as above, we assume that $N=\nabla \phi$ for some $\phi$ of class $C^{2}$.

(H2) There exists $\gamma>1$ such that $\forall u \in H, \forall t \in[0,1], \phi(t u) \geq t^{\gamma+1} \phi(u)$,

(H3) $\phi$ is convex and $\forall u \in H,\langle N(u), u\rangle \geq 2 \phi(u)$,

(H4) $\exists \kappa, \bar{\alpha}, \bar{\beta} \geq 0$ such that $\bar{\alpha}<2, \bar{\alpha}+2 \bar{\beta}>2$ and $\phi(u) \leq \kappa\|u\|^{\bar{\alpha}}\langle A u, u\rangle^{\bar{\beta}}$ for all $u \in H$,

(H5) $N$ is a compact operator,

(H6) there exist a sequence $\left(v_{n}\right) \subset H$ and a constant $M>0$ such that

$-\forall n \in \mathbb{N}\left\langle A v_{n}, v_{n}\right\rangle=1$,

$-\left(\phi\left(v_{n}\right)\right)$ is bounded,

- $\forall n \in \mathbb{N}\left\|(L-l A) v_{n}\right\|^{2} \leq M\left|\left\langle(L-l A) v_{n}, v_{n}\right\rangle\right|$,

$-\forall n \in \mathbb{N} \phi\left(v_{n}\right) \neq 0$ and $\lim _{n \rightarrow \infty} \frac{\left\langle(L-l A) v_{n}, v_{n}\right\rangle}{\phi\left(v_{n}\right)}=0$.

Hypothesis (H6) was introduced by Heinz, Küpper and Stuart $[10,11]$ in their theory of bifurcation from the essential spectrum.

We will then prove an abstract version of Theorem 1. But first consider the following (non penalized) functional:

$$
J_{\infty}(u)=\frac{1}{2}\langle L u, u\rangle-\phi(u)
$$

for $u \in H$. We can, as for $J_{r, \mu}$, make a variational reduction of $J_{\infty}$, leading to a reduction map $g_{\infty}: H_{+} \rightarrow H_{-}$and to a reduced functional $F_{\infty}: H_{+} \rightarrow H_{-}$satisfying

$$
F_{\infty}(v)=J_{\infty}\left(v+g_{\infty}(v)\right)=\max \left\{J_{\infty}(v+w): w \in H_{-}\right\} .
$$


Moreover, for all $\hat{v} \in H_{+} \backslash\{0\}$,

$$
c_{\infty} \leq \sup _{0 \leq t<\langle A \hat{v}, \hat{v}\rangle^{-1 / 2}} F_{\infty}(t \hat{v}) .
$$

Theorem 7. Under hypotheses (H1) to (H6), the following alternative holds:

1. there exists $u \in H \backslash\{0\}$ and $\lambda \in(-m, l)$ such that

$$
L u-N(u)=\lambda A u, \quad\langle A u, u\rangle=1,
$$

2. or, for all $\lambda \in(-m, l)$, there exists $u_{\lambda} \in H \backslash\{0\}$ such that

$$
L u_{\lambda}-N\left(u_{\lambda}\right)=\lambda A u_{\lambda}, \quad\left\langle A u_{\lambda}, u_{\lambda}\right\rangle<1 .
$$

In the second case, $\lim \sup _{\lambda \rightarrow-m} \phi\left(P u_{\lambda}\right)>0$ and $\lim \sup _{\lambda \rightarrow-m}\left\|u_{\lambda}\right\|<\infty$.

The second case cannot occur if, in addition, $2^{\bar{\alpha}+2 \bar{\beta}} \kappa\|A\|^{1-\bar{\alpha} / 2}<\delta$, that is, if the nonlinearity is "small" enough.

Finally, if the additional assumption

(H7) If $u_{n} \rightarrow u_{\infty}$ weakly in $H$, then $N\left(u_{n}\right) \rightarrow N\left(u_{\infty}\right)$ and $\phi\left(u_{n}\right) \rightarrow \phi\left(u_{\infty}\right)$

holds and if there is no solution to

$$
L u-N(u)=\lambda A u, \quad\langle A u, u\rangle=1,
$$

for any $\lambda \in(-m, l)$, then we can find $u_{m} \in H \backslash\{0\}$ such that

$$
L u_{m}-N\left(u_{m}\right)=-m A u_{m}, \quad\left\langle A u_{m}, u_{m}\right\rangle \leq 1 .
$$

\section{Proof. Preliminary remarks.}

If hypotheses (H1) to (H6) hold, they also hold when $L$ is replaced by $L-s A$ and $l$ by $l-s$, for all $s \in(-m, l)$. Hence it suffices to check the assumptions of Theorem 5 for $s=0$ and then the statement of the alternative is a direct consequence of Corollary 6.

Hypothesis (H3) implies that $\phi \geq 0$ (because $\phi(0)=0$ and $\left.\phi^{\prime}(0)=0\right)$ and $(\mathrm{C} 1)$ is then obvious. Hypothesis (H4) implies that $\phi^{\prime \prime}(0)=N^{\prime}(0)=0$, and thus (C2) holds. Moreover $g_{r, \mu}^{\prime}(0)=0$. Indeed

$$
L\left(g_{r, \mu}^{\prime}(0) v\right)=(I-P) N^{\prime}(0)\left(v+g_{r, \mu}^{\prime}(0) v\right)+2 f_{r}^{\prime}(0) A_{\mu}\left(g_{r, \mu}^{\prime}(0) v\right)=0
$$

and $g_{r, \mu}^{\prime}(0) v=0$ for all $v \in H_{+}$. Therefore $F_{r, \mu}^{\prime \prime}=P L$.

Step 1. Proof of the inequality $c_{\infty}<l / 2$.

This result is an easy consequence of results originally due to Heinz, Küpper and Stuart $[10,11]$. Indeed, they proved that hypothesis (H6) implies the existence of a bounded sequence $\left(z_{n}\right) \subset H_{+}$such that

$$
\forall n \in \mathbb{N}\left\langle A z_{n}, z_{n}\right\rangle=1, \quad \forall n \in \mathbb{N} \phi\left(z_{n}\right) \neq 0 \text { and } \lim _{n \rightarrow \infty} \frac{\left\langle(L-l A) z_{n}, z_{n}\right\rangle}{\phi\left(z_{n}\right)}=0
$$


See [3] for the proof rewritten with notations close to ours.

Then, we proceed as follows. We first assume that there exists a sequence $\left(\hat{v}_{n}\right) \subset H_{+} \backslash\{0\}$ such that

$$
F_{\infty}\left(\hat{v}_{n}\right)<\frac{l}{2}\left\langle A \hat{v}_{n}, \hat{v}_{n}\right\rangle \forall n \in \mathbb{N} \text { and } \lim _{n \rightarrow \infty}\left\|\hat{v}_{n}\right\|=0,
$$

and prove

$$
c_{\infty}<\frac{l}{2} .
$$

Indeed, let us choose $n$ so large that $t \rightarrow F_{\infty}\left(t \hat{v}_{n}\right)$ is increasing for $0 \leq t \leq 1$ and $\left\langle A \hat{v}_{n}, \hat{v}_{n}\right\rangle<1$. Hypothesis (H3) implies that $t \rightarrow t^{-2} \phi(t x)$ is non-decreasing for all $x \in H$, so that, for $1 \leq t \leq\left\langle A \hat{v}_{n}, \hat{v}_{n}\right\rangle^{-1 / 2}$, we get $\phi\left(t \hat{v}_{n}+g_{\infty}\left(t \hat{v}_{n}\right)\right) \geq$ $t^{2} \phi\left(\hat{v}_{n}+t^{-1} g_{\infty}\left(t \hat{v}_{n}\right)\right)$ and

$$
\begin{aligned}
F_{\infty}\left(t \hat{v}_{n}\right)=J_{\infty}\left(t \hat{v}_{n}+g_{\infty}\left(t \hat{v}_{n}\right)\right) \leq t^{2} J_{\infty}\left(\hat{v}_{n}+t^{-1} g_{\infty}\left(t \hat{v}_{n}\right)\right) & \\
& \stackrel{(4.27)}{\leq} t^{2} F_{\infty}\left(\hat{v}_{n}\right)<t^{2} \frac{l}{2}\left\langle A \hat{v}_{n}, \hat{v}_{n}\right\rangle \leq \frac{l}{2} .
\end{aligned}
$$

Hence, by the above estimates and (4.28),

$$
c_{\infty} \leq \sup _{0 \leq t<\left\langle A \hat{v}_{n}, \hat{v}_{n}\right\rangle^{-1 / 2}} F_{\infty}\left(t \hat{v}_{n}\right)<\frac{l}{2}
$$

for some fixed $n$ chosen large enough, so that (4.30) holds true.

We now construct the sequence $\left(\hat{v}_{n}\right)$ from the sequence $\left(z_{n}\right)$ given by $(4.29)$ as follows. We set $\hat{v}_{n}=t_{n} z_{n}$ with $\lim _{n \rightarrow \infty} t_{n}=0^{+}$, and get for $n$ large enough

$$
\begin{aligned}
F_{\infty}\left(\hat{v}_{n}\right) \quad= & \frac{1}{2}\left\langle L\left(\hat{v}_{n}+g_{\infty}\left(\hat{v}_{n}\right)\right), \hat{v}_{n}+g_{\infty}\left(\hat{v}_{n}\right)\right\rangle-\phi\left(\hat{v}_{n}+g_{\infty}\left(\hat{v}_{n}\right)\right) \\
\stackrel{(H 3)}{\leq} & \frac{1}{2}\left\langle L\left(\hat{v}_{n}+g_{\infty}\left(\hat{v}_{n}\right)\right), \hat{v}_{n}+g_{\infty}\left(\hat{v}_{n}\right)\right\rangle-2 \phi\left(\hat{v}_{n} / 2\right)+\phi\left(-g_{\infty}\left(\hat{v}_{n}\right)\right) \\
\stackrel{(H 2)}{\leq} & \frac{1}{2}\left\langle L\left(\hat{v}_{n}+g_{\infty}\left(\hat{v}_{n}\right)\right), \hat{v}_{n}+g_{\infty}\left(\hat{v}_{n}\right)\right\rangle-2^{-\gamma} \phi\left(\hat{v}_{n}\right)+\phi\left(-g_{\infty}\left(\hat{v}_{n}\right)\right) \\
\stackrel{(3.11),(H 4)}{\leq} & \frac{1}{2}\left\langle L \hat{v}_{n}, \hat{v}_{n}\right\rangle-\frac{\delta}{2}\left\|g_{\infty}\left(\hat{v}_{n}\right)\right\|^{2}-2^{-\gamma} \phi\left(\hat{v}_{n}\right)+\kappa\|A\|^{\bar{\beta}}\left\|g_{\infty}\left(\hat{v}_{n}\right)\right\|^{\bar{\alpha}+2 \bar{\beta}} \\
\stackrel{\bar{\alpha}+2 \bar{\beta}>2}{\leq} & \frac{1}{2}\left\langle L \hat{v}_{n}, \hat{v}_{n}\right\rangle-2^{-\gamma} \phi\left(\hat{v}_{n}\right) \stackrel{(H 2)}{\leq} t_{n}^{2} \frac{1}{2}\left\langle L z_{n}, z_{n}\right\rangle-2^{-\gamma} t_{n}^{1+\gamma} \phi\left(z_{n}\right) \\
\text { see below } & \frac{l}{2} t_{n}^{2}\left\langle A z_{n}, z_{n}\right\rangle=\frac{l}{2}\left\langle A \hat{v}_{n}, \hat{v}_{n}\right\rangle
\end{aligned}
$$

if

$$
\frac{\left\langle(L-l A) z_{n}, z_{n}\right\rangle}{\phi\left(z_{n}\right)}<2^{1-\gamma} t_{n}^{\gamma-1}
$$

which holds for $t_{n}$ defined by

$$
t_{n}=2\left\{2^{\gamma-1} \frac{\left\langle(L-l A) z_{n}, z_{n}\right\rangle}{\phi\left(z_{n}\right)}\right\}^{1 /(\gamma-1)} \underset{n \rightarrow+\infty}{\longrightarrow} 0 .
$$


Step 2. For $r>2$ and $\mu>0$ fixed, existence of relatively compact mountain-pass critical sequences satisfying condition (3.20), as a consequence of the inequality $c_{\infty}<l / 2$.

Let $\xi_{r}, \tau_{r} \in(0,1)$ be such that

$$
c_{\infty}=f_{r}^{\prime}\left(\xi_{r}\right) \xi_{r}-f_{r}\left(\xi_{r}\right) \text { and } 2 f_{r}^{\prime}\left(\tau_{r}\right)=l,
$$

and set $d_{r}=f_{r}^{\prime}\left(\tau_{r}\right) \tau_{r}-f_{r}\left(\tau_{r}\right)$. By the properties of $f_{r}$ we have

$$
\xi_{r}, \tau_{r} \underset{r \rightarrow+\infty}{\longrightarrow} \quad 1, \quad d_{r} \underset{r \rightarrow+\infty}{\longrightarrow} \quad l / 2,
$$

$c_{\infty}<d_{r}$ for $r$ large (because $\left.c_{\infty}<l / 2\right), \xi_{r}<\tau_{r}$ (because $s \rightarrow f_{r}^{\prime}(s) s-f_{r}(s)$ is increasing) and, still for $r$ large,

$$
\begin{gathered}
d_{r}-c_{\infty}=f_{r}^{\prime}\left(\tau_{r}\right) \tau_{r}-f_{r}\left(\tau_{r}\right)- \\
f_{r}^{\prime}\left(\xi_{r}\right) \xi_{r}+f_{r}\left(\xi_{r}\right)=\int_{\xi_{r}}^{\tau_{r}} f_{r}^{\prime \prime}(s) s d s \\
\leq \int_{\xi_{r}}^{\tau_{r}} f_{r}^{\prime \prime}(s) d s=(l / 2)-f_{r}^{\prime}\left(\xi_{r}\right) .
\end{gathered}
$$

Hence, by Step 1,

$$
\limsup _{r \rightarrow+\infty} f_{r}^{\prime}\left(\xi_{r}\right) \leq c_{\infty}<l / 2 .
$$

Moreover for $\mu>0$ we also define $\sigma_{r, \mu} \in(0,1)$ by $c_{r, \mu}=f_{r}^{\prime}\left(\sigma_{r, \mu}\right) \sigma_{r, \mu}-f_{r}\left(\sigma_{r, \mu}\right)$.

Let us now consider a (mountain-pass) critical sequence of $F_{r, \mu}$ at level $c_{r, \mu}$, denoted by $\left(v_{n}\right)$. It is well known that such sequences exist at a mountain-pass level. Defining $u_{n}=v_{n}+g_{r, \mu}\left(v_{n}\right), \lambda_{n}=2 f_{r}^{\prime}\left(\left\langle A_{\mu} u_{n}, u_{n}\right\rangle\right)$ and adding $\left\langle\nabla F_{r, \mu}\left(v_{n}\right), v_{n}\right\rangle=$ $\left\langle\nabla J_{r, \mu}\left(u_{n}\right), v_{n}\right\rangle$ and $\left\langle\nabla J_{r, \mu}\left(u_{n}\right), w_{n}\right\rangle=0$ (see (3.14) and (3.16)), we get that

$$
\begin{aligned}
\left\langle\nabla F_{r, \mu}\left(v_{n}\right), v_{n}\right\rangle= & \left\langle\nabla J_{r, \mu}\left(u_{n}\right), u_{n}\right\rangle \\
& =\left\langle L u_{n}, u_{n}\right\rangle-\left\langle N\left(u_{n}\right), u_{n}\right\rangle-\lambda_{n}\left\langle A_{\mu} u_{n}, u_{n}\right\rangle=\epsilon_{n}\left\|v_{n}\right\|,
\end{aligned}
$$

for some sequence $\epsilon_{n} \rightarrow 0$, because $\nabla F_{r, \mu}\left(v_{n}\right) \rightarrow 0$. By (H3),

$$
\begin{array}{cl} 
& \lambda_{n}\left\langle A_{\mu} u_{n}, u_{n}\right\rangle-2 f_{r}\left(\left\langle A_{\mu} u_{n}, u_{n}\right\rangle\right) \\
\leq & \lambda_{n}\left\langle A_{\mu} u_{n}, u_{n}\right\rangle-2 f_{r}\left(\left\langle A_{\mu} u_{n}, u_{n}\right\rangle\right)+\left\langle N\left(u_{n}\right), u_{n}\right\rangle-2 \phi\left(u_{n}\right) \\
\stackrel{(4.32)}{=} & \left\langle L u_{n}, u_{n}\right\rangle-\left\langle N\left(u_{n}\right), u_{n}\right\rangle-\epsilon_{n}\left\|v_{n}\right\|-2 f_{r}\left(\left\langle A_{\mu} u_{n}, u_{n}\right\rangle\right) \\
& +\left\langle N\left(u_{n}\right), u_{n}\right\rangle-2 \phi\left(u_{n}\right)=2 J_{r, \mu}\left(u_{n}\right)-\epsilon_{n}\left\|v_{n}\right\| .
\end{array}
$$

It follows that

$$
\begin{aligned}
& \lim _{n \rightarrow \infty}\left\{f_{r}^{\prime}\left(\left\langle A_{\mu} u_{n}, u_{n}\right\rangle\right)\right.\left.\left\langle A_{\mu} u_{n}, u_{n}\right\rangle-f_{r}\left(\left\langle A_{\mu} u_{n}, u_{n}\right\rangle\right)\right\} \leq \lim _{n \rightarrow \infty} J_{r, \mu}\left(u_{n}\right) \\
&=c_{r, \mu}=f_{r}^{\prime}\left(\sigma_{r, \mu}\right) \sigma_{\mu}-f_{r}\left(\sigma_{r, \mu}\right) \leq c_{\infty}=f_{r}^{\prime}\left(\xi_{r}\right) \xi_{r}-f_{r}\left(\xi_{r}\right) .
\end{aligned}
$$

Thus, by the strict monotonicity of $s \mapsto f_{r}(s)$ and of $s \mapsto f_{r}^{\prime}(s) s-f_{r}(s)$, we get

$$
\limsup _{n \rightarrow \infty} \lambda_{n}=2 \limsup _{n \rightarrow \infty} f_{r}^{\prime}\left(\left\langle A_{\mu} u_{n}, u_{n}\right\rangle\right) \leq 2 f_{r}^{\prime}\left(\xi_{r}\right)
$$


and by (4.31),

$$
\limsup _{n \rightarrow \infty} \lambda_{n}=2 \limsup _{n \rightarrow \infty} f_{r}^{\prime}\left(\left\langle A_{\mu} u_{n}, u_{n}\right\rangle\right) \leq 2 c_{\infty}<l,
$$

that is, (3.20) holds true.

The relative compactness of $\left(u_{n}\right)$ follows from boundedness of $\left(u_{n}\right)$ (see (3.19)), the compactness of $N$ (Hypothesis (H5)), the inequalities $0 \leq \lim \sup _{n \rightarrow \infty} \lambda_{n} \leq$ $2 c_{\infty}<l$ and from

$$
L u_{n}-N\left(u_{n}\right)-\lambda_{n} A_{\mu} u_{n} \rightarrow 0 \text { in } H,
$$

provided $\mu$ is small enough so that

$$
\limsup _{n \rightarrow \infty} \lambda_{n}\left\langle l_{\mu}:=\inf _{u \in H_{+} \backslash\{0\}} \frac{\langle L u, u\rangle}{\left\langle A_{\mu} u, u\right\rangle}=\frac{l}{1+\mu l} .\right.
$$

Step 3. Compactness holds for critical sequences satisfying (3.21).

Let $r_{n}>2, \mu_{n}>0$ be such that $\left(r_{n}, \mu_{n}\right)$ converges to $(\infty, 0)$ and let $\left(v_{n}\right)$ be a sequence such that

$$
F_{r_{n}, \mu_{n}}\left(v_{n}\right)=c_{r_{n}, \mu_{n}}, \quad \nabla F_{r_{n}, \mu_{n}}\left(v_{n}\right)=0(\forall n \in \mathbb{N}) .
$$

Note that the domain of $F_{r_{n}, \mu_{n}}$ depends on $n$.

Define now $u_{n}=v_{n}+g_{r_{n}, \mu_{n}}\left(v_{n}\right)$ and $\lambda_{n}=2 f_{r_{n}}^{\prime}\left(\left\langle A_{\mu_{n}} u_{n}, u_{n}\right\rangle\right)$. We have

$$
L u_{n}=N\left(u_{n}\right)+\lambda_{n} A_{\mu_{n}} u_{n}, \quad \lambda_{n} \leq l^{\prime}<l .
$$

Setting $v_{n}=P u_{n}$ and $w_{n}=(I-P) u_{n}$, we obtain

$$
\begin{aligned}
\delta\left\|u_{n}\right\|^{2}-l \stackrel{(3.11)}{\leq} & \left\langle L v_{n}, v_{n}\right\rangle-\left\langle L w_{n}, w_{n}\right\rangle-\lambda_{n}\left\langle A_{\mu_{n}} v_{n}, v_{n}\right\rangle+\lambda_{n}\left\langle A_{\mu_{n}} w_{n}, w_{n}\right\rangle \\
& =\quad\left\langle\left(L-\lambda_{n} A_{\mu_{n}}\right)\left(v_{n}+w_{n}\right), v_{n}-w_{n}\right\rangle \\
& =\left\langle N\left(u_{n}\right), v_{n}-w_{n}\right\rangle=\left\langle N\left(u_{n}\right), 2 v_{n}-u_{n}\right\rangle \\
& \stackrel{(H 3)}{\leq} \\
& \phi\left(2 v_{n}\right)-\phi\left(u_{n}\right) \stackrel{(H 4)}{\leq} 2^{\bar{\alpha}+2 \bar{\beta}} \kappa\left\|u_{n}\right\|^{\bar{\alpha}}
\end{aligned}
$$

with $0 \leq \bar{\alpha}<2$. Hence, since $\bar{\alpha}<2$, we have the useful estimate

$$
\limsup _{n \rightarrow+\infty}\left\|u_{n}\right\| \leq C \text {, where } C \text { depends only on } l, \delta, \bar{\alpha}, \bar{\beta}, \kappa \text {. }
$$

The relative compactness of $\left(u_{n}\right)$ follows, as previously, from (4.34), the compactness of $N$, the inequalities $0 \leq \lim \sup _{n \rightarrow \infty} \lambda_{n}<l$ and $\mu_{n} \rightarrow 0$.

\section{Step 4. End of the proof of Theorem 7 .}

The first part of the theorem is a direct consequence of Theorem 5, Corollary 6 and Steps 1 and 2. 
Let us assume that the second case of the alternative occurs. Writing $w_{\lambda}=$ $(I-P) u_{\lambda}$ and $v_{\lambda}=P u_{\lambda}$, we get $\lim \sup _{\lambda \rightarrow-m} \phi\left(2 v_{\lambda}\right)>0$ by the following standard argument. If not, we would have for $\lambda \in(-m, 0)$

$$
\begin{aligned}
& \delta\left\|v_{\lambda}\right\|^{2}+(\lambda+m)\left\langle A w_{\lambda}, w_{\lambda}\right\rangle \\
& \stackrel{(3.11),(3.13)}{\leq}\left\langle L v_{\lambda}, v_{\lambda}\right\rangle+\lambda\left\langle A w_{\lambda}, w_{\lambda}\right\rangle-\left\langle L w_{\lambda}, w_{\lambda}\right\rangle \\
& \stackrel{\lambda<0}{\leq} \quad\left\langle L v_{\lambda}, v_{\lambda}\right\rangle+\lambda\left\langle A w_{\lambda}, w_{\lambda}\right\rangle-\left\langle L w_{\lambda}, w_{\lambda}\right\rangle-\lambda\left\langle A v_{\lambda}, v_{\lambda}\right\rangle \\
& =\left\langle L\left(v_{\lambda}+w_{\lambda}\right), v_{\lambda}-w_{\lambda}\right\rangle+\lambda\left\langle A\left(w_{\lambda}+v_{\lambda}\right), w_{\lambda}-v_{\lambda}\right\rangle \\
& =\left\langle N\left(u_{\lambda}\right)+\lambda A u_{\lambda}, v_{\lambda}-w_{\lambda}\right\rangle+\lambda\left\langle A u_{\lambda}, w_{\lambda}-v_{\lambda}\right\rangle \\
& =\left\langle N\left(u_{\lambda}\right), v_{\lambda}-w_{\lambda}\right\rangle=\left\langle N\left(u_{\lambda}\right), 2 v_{\lambda}-u_{\lambda}\right\rangle \\
& \stackrel{(H 3)}{\leq} \quad \phi\left(2 v_{\lambda}\right)-\phi\left(u_{\lambda}\right) \leq \phi\left(2 v_{\lambda}\right) \\
& \stackrel{(H 4)}{\leq} \quad 2^{\bar{\alpha}+2 \bar{\beta}} \kappa\left\langle A v_{\lambda}, v_{\lambda}\right\rangle^{\bar{\beta}}\left\|v_{\lambda}\right\|^{\bar{\alpha}} \\
& \leq \quad 2^{\bar{\alpha}+2 \bar{\beta}} \kappa\|A\|^{\bar{\beta}}\left\|v_{\lambda}\right\|^{\bar{\alpha}+2 \bar{\beta}},
\end{aligned}
$$

and $\lim _{\lambda \rightarrow-m}\left\|v_{\lambda}\right\|=0$ (because $\lim _{\lambda \rightarrow-m} \phi\left(2 v_{\lambda}\right)=0$ by assumption). Hence

$$
\delta\left\|v_{\lambda}\right\|^{2}+(\lambda+m)\left\langle A w_{\lambda}, w_{\lambda}\right\rangle \leq 2^{\bar{\alpha}+2 \bar{\beta}} \kappa\|A\|^{\bar{\beta}}\left\|v_{\lambda}\right\|^{\bar{\alpha}+2 \bar{\beta}} \leq \frac{\delta}{2}\left\|v_{\lambda}\right\|^{2}
$$

for all $\lambda$ near enough to $-m$, which leads to the contradiction $v_{\lambda}=w_{\lambda}=u_{\lambda}=0$.

Analogously, we get

$$
\begin{aligned}
\delta\left\|u_{\lambda}\right\|^{2}-m & =\delta\left\|v_{\lambda}\right\|^{2}+\delta\left\|w_{\lambda}\right\|^{2}-m \\
& \leq\left\langle L v_{\lambda}, v_{\lambda}\right\rangle-\left\langle L w_{\lambda}, w_{\lambda}\right\rangle+\lambda\left\langle A w_{\lambda}, w_{\lambda}\right\rangle \\
& \leq\left\langle L v_{\lambda}, v_{\lambda}\right\rangle-\left\langle L w_{\lambda}, w_{\lambda}\right\rangle+\lambda\left\langle A w_{\lambda}, w_{\lambda}\right\rangle-\lambda\left\langle A v_{\lambda}, v_{\lambda}\right\rangle \\
& \leq 2^{\bar{\alpha}+2 \bar{\beta}} \kappa\left\langle A v_{\lambda}, v_{\lambda}\right\rangle^{\bar{\beta}}\left\|v_{\lambda}\right\|^{\bar{\alpha}} \\
& \leq 2^{\bar{\alpha}+2 \bar{\beta}} \kappa\left\|u_{\lambda}\right\|^{\bar{\alpha}}
\end{aligned}
$$

and therefore $\lim \sup _{\lambda \rightarrow-m}\left\|u_{\lambda}\right\|<\infty$ (thanks to $\bar{\alpha}<2$ ).

We also obtain

$$
\delta\left\|v_{\lambda}\right\|^{2}+(\lambda+m)\left\langle A w_{\lambda}, w_{\lambda}\right\rangle \leq 2^{\bar{\alpha}+2 \bar{\beta}} \kappa\|A\|^{1-\bar{\alpha} / 2}\left\|v_{\lambda}\right\|^{2}
$$

and the same kind of argument shows that the second case of the alternative cannot occur if

$$
2^{a+2 \bar{\beta}} \kappa\|A\|^{1-\bar{\alpha} / 2}<\delta .
$$

Finally, to prove the last statement in the theorem, that is, the passage $\lambda \rightarrow-m$ in the second case of the above alternative, we first note that $\lim \sup _{\lambda \rightarrow-m}\left\|u_{\lambda}\right\|<$ $\infty$ and that there exists a sequence $\lambda_{n} \rightarrow-m$ and $u \in H$ such that $u_{\lambda_{n}} \rightarrow u$, $L u_{\lambda_{n}} \rightarrow L u, A u_{\lambda_{n}} \rightarrow A u$. Hence from (H7), N( $\left.u_{\lambda_{n}}\right) \rightarrow N(u), \phi\left(u_{\lambda_{n}}\right) \rightarrow \phi(u)$, $L u-N(u)=-m A u$ and $\langle A u, u\rangle \leq 1$. If $u=0$, then $P u_{\lambda_{n}} \rightarrow 0$ and $\phi\left(2 P u_{\lambda_{n}}\right) \rightarrow 0$, which has been proved to be impossible above. 
We will now deal with the particular equation (1.1) and prove what we stated in the introduction.

Proof of Theorem 1. Although Theorem 1 is stated for $d \geq 2$, let us also mention the case $d=1$. In (2.6), we suppose without loss of generality that the spectrum $\sigma(-\Delta+p)$ has a gap $(-m, l) \ni 0$, that is, $l, m>0, \sigma(-\Delta+p)$ does not intersect $(-m, l)$ and $\{l,-m\} \subset \sigma(-\Delta+p)$. This can be achieved by replacing $p$ by $p-\lambda_{0}$ (see the Introduction for the meaning of $\lambda_{0}$ ).

We set $H=H^{1}\left(\mathbb{R}^{d}\right)$ endowed with the inner-product defined in (2.7),

$$
\langle L u, v\rangle_{H 1}:=\int_{\mathbb{R}^{d}}(\nabla u \cdot \nabla v+p u v) d x \text { and }\langle A u, v\rangle_{H 1}:=\int_{\mathbb{R}^{d}} u v d x \quad \forall u, v \in H,
$$

which defines $L: H \rightarrow H$ and $A: H \rightarrow H$ uniquely. We also set

$$
\phi(u)=\frac{1}{1+\gamma} \int_{\mathbb{R}^{d}} a(x)|u(x)|^{1+\gamma} d x \quad \forall u \in H .
$$

Since $a \in L^{\infty}\left(\mathbb{R}^{d}\right)$, hypothesis (H4) is satisfied provided that $\gamma<3$, if $d=1,2$, or $\gamma<1+\frac{4}{d}$, if $d \geq 3$. Note that for $d \geq 2$ and any $q>\frac{2}{1+\frac{4}{d}-\gamma}$, the condition $a \in L^{q}\left(\mathbb{R}^{d}\right)$ implies (H4) with constants $\bar{\alpha}, \bar{\beta}, \kappa$ depending on $q$. (H5) holds because of (2.8). (H2) and (H3) also hold true.

By results of Heinz, Küpper and Stuart [10, 11], hypothesis (H6) holds when $d \geq 2$ because, as assumed in the theorem, for $|x|$ large, $a(x) \geq C|x|^{-\tau}$, for some $\tau<\tau(\gamma), \tau(\gamma)=2-\frac{d}{2}(\gamma-1)$. Note that for $d=1$ there is no assumption on the speed of decay of $a$ at infinity, but $p$ is assumed piecewise continuous and $a$ continuous (see condition (C4), Corollary 5.3 and Theorem 5.4 in [14]).

Proof of Theorem 2. In the case $d=1$, by a theorem of Rofe-Beketof [16, 13], the equation

$$
-u^{\prime \prime}(x)+\{p(x)+m\} u(x)-a(x)|u(x)|^{\gamma-1} u(x)=0 \text { in } \mathbb{R}, \quad 0<\int_{\mathbb{R}^{d}} u^{2}(x) d x \leq 1
$$

seen as a linear equation for $u$ in which $a(x)|u|^{\gamma-1}$ is a coefficient, has no solution if

$$
\int_{\mathbb{R}}|x| a(x)|u(x)|^{\gamma-1} d x<\infty
$$

Since $|u|^{\gamma-1} \in L^{2 /(\gamma-1)}(\mathbb{R}),(4.36)$ holds if $\gamma<3$ and $|\cdot| a(\cdot) \in L^{2 /(3-\gamma)}(\mathbb{R})$. See Theorem 3.2 in [14], where (4.36) is used in an analogous discussion.

Proof of Theorem 3. This is a direct consequence of Theorem 7: when $\|a\|_{L^{q}\left(\mathbb{R}^{d}\right)}$ is small enough, then so is $2^{\bar{\alpha}+2 \bar{\beta}} \kappa\|A\|^{1-\bar{\alpha} / 2}$ (see the remark made in the proof of Theorem 2 about the dependence on $q$ of $\kappa, \bar{\alpha}$ and $\bar{\beta}$ ). 


\section{Proof of Theorem 4.}

In order to prove this theorem, we notice that the assumptions (C1) and (C2) are implied by (1.5). Indeed remember that

$$
\phi(u)=\frac{\alpha}{2} \int_{\mathbb{R}^{d}} \frac{|u|^{2}}{|x|} d x-\frac{\beta}{4} \iint_{\mathbb{R}^{d} \times \mathbb{R}^{d}} \frac{u^{2}(x) u^{2}(y)}{|x-y|} d x d y,
$$

so that

$$
\begin{aligned}
\phi^{\prime \prime}(u)(z, z)=\alpha \int_{\mathbb{R}^{d}} \frac{|z|^{2}}{|x|} d x-\beta \iint_{\mathbb{R}^{d} \times \mathbb{R}^{d}} \frac{u^{2}(x) z^{2}(y)}{|x-y|} d x d y \\
-2 \beta \iint_{\mathbb{R}^{d} \times \mathbb{R}^{d}} \frac{u(x) z(x) u(y) z(y)}{|x-y|} d x d y .
\end{aligned}
$$

Also note that

$$
\begin{aligned}
& \mid \iint_{\mathbb{R}^{d} \times \mathbb{R}^{d}} \frac{u(x) z(x) u(y) z(y)}{|x-y|} d x d y \mid \\
& \leq\left\{\iint_{\mathbb{R}^{d} \times \mathbb{R}^{d}} \frac{u^{2}(x) z^{2}(y)}{|x-y|} d x d y \iint_{\mathbb{R}^{d} \times \mathbb{R}^{d}} \frac{z^{2}(x) u^{2}(y)}{|x-y|} d x d y\right\}^{1 / 2} \\
& \quad=\iint_{\mathbb{R}^{d} \times \mathbb{R}^{d}} \frac{u^{2}(x) z^{2}(y)}{|x-y|} d x d y .
\end{aligned}
$$

Next, we will prove that the other assumptions of Theorem 5 hold true. For simplicity, we assume $\lambda_{0}=0$.

First Step : Condition (3.20) holds true.

Let $(r, \mu) \in(2,+\infty) \times[0,1)$ and let $\left(v_{n}\right)$ be a sequence in $H_{+}$such that

$$
\nabla F_{r, \mu}\left(v_{n}\right) \rightarrow 0, F_{r, \mu}\left(v_{n}\right) \rightarrow c_{r, \mu} .
$$

Assume, moreover, that there is a sequence $\delta_{n} \rightarrow 0$ such that the quadratic form

$$
H_{+} \ni z \rightarrow F_{r, \mu}^{\prime \prime}\left(v_{n}\right) \cdot z^{2}+\delta_{n}\|z\|_{H^{1}\left(\mathbb{R}^{d}\right)}^{2}
$$

has a negative space of dimension at most 1 . It is known that such mountain-pass critical sequences with "Morse-type information" exist, by Theorems 4.1 and 6.2 in [9]. Let us set

$$
u_{n}=v_{n}+g_{r, \mu}\left(v_{n}\right), \quad \lambda_{n}=2 f_{r}^{\prime}\left(\left\langle A_{\mu} u_{n}, u_{n}\right\rangle\right) .
$$

We may assume, after extraction, that $\left(\lambda_{n}\right)$ has a limit $\lambda_{*}$. This limit is finite, since for $r, \mu$ fixed the sequence $\left(u_{n}\right)$ stays away from the boundary of $U_{\mu}$. Since $\left(u_{n}\right)$ is bounded in $H^{1}$ (see (3.19)), we may also assume, after a new extraction, that this sequence has a weak limit $u_{*}$ in $H^{1}$. Using the information $\nabla F_{r, \mu}\left(v_{n}\right) \rightarrow_{n} 0$, one easily shows that $u_{*}$ is in fact a limit for the $H_{l o c}^{1}$ topology. Then $u_{*}$ solves $L u_{*}-N\left(u_{*}\right)=\lambda_{*} A_{\mu} u_{*}$ and $\left\langle A_{\mu} u_{*}, u_{*}\right\rangle<1$. 
Our goal now is to study the second derivative $F_{r, \mu}^{\prime \prime}\left(v_{n}\right)$.

Following [11], let us consider a function $\psi \in H_{l o c}^{2}\left(\mathbb{R}^{d}\right) \cap C^{1}\left(\mathbb{R}^{d}\right)$ which is a nontrivial solution of $-\Delta u+p u=l u$. We can choose it to be uniformly almostperiodic in the sense of Besicovich [2], and such that $\psi^{2}$ is periodic. Then we consider $f \in C_{0}^{\infty}\left(\mathbb{R}^{d}\right)$ such that $\|f\|_{L^{2}\left(\mathbb{R}^{d}\right)}=1$ and for every $\sigma>0$ we define $k_{\sigma}(x):=\sigma^{-N / 2} f\left(\frac{x}{\sigma}\right) \psi(x)$.

By the Riemann-Lebesgue lemma (see [11]), we have

$$
\begin{aligned}
& \left((-\Delta+p(x)-l) k_{\sigma}, k_{\sigma}\right)_{L^{2}\left(\mathbb{R}^{d}\right)} \\
= & \int_{\mathbb{R}^{d}}\left\{\sigma^{-2} \Delta f(x) \psi(\sigma x)+2 \sigma^{-1} \nabla f(x) \cdot \nabla \psi(\sigma x)\right\} f(x) \psi(\sigma x) d x \\
= & \int_{\mathbb{R}^{d}} \sigma^{-2} f(x) \Delta f(x) \psi^{2}(\sigma x)+(1 / 2) \sigma^{-2} \nabla\left(f^{2}(x)\right) \cdot \nabla\left(\psi^{2}(\sigma x)\right) d x \\
= & \int_{\mathbb{R}^{d}} \sigma^{-2} f(x) \Delta f(x) \psi^{2}(\sigma x)-(1 / 2) \sigma^{-2} \Delta\left(f^{2}(x)\right) \psi^{2}(\sigma x) d x \\
= & \sigma^{-2} M\left(\psi^{2}\right) \int_{\mathbb{R}^{d}} f(x) \Delta f(x)-(1 / 2) \Delta\left(f^{2}(x)\right) d x+o\left(\sigma^{-2}\right)=O\left(\frac{1}{\sigma^{2}}\right),
\end{aligned}
$$

where $M\left(\psi^{2}\right)$ denotes the mean-value of $\psi^{2}$. By the same computations,

$$
\left\|(-\Delta+p(x)-l) k_{\sigma}\right\|_{L^{2}\left(\mathbb{R}^{d}\right)}=O\left(\sigma^{-1}\right) .
$$

So, setting $P k_{\sigma}:=k_{\sigma}^{+}$and $(I-P) k_{\sigma}:=k_{\sigma}^{-}$, we get

Const $\left\|k_{\sigma}^{-}\right\|_{H^{1}\left(\mathbb{R}^{d}\right)}^{2} \leq\left((-\Delta+p-l) k_{\sigma}, k_{\sigma}-2 k_{\sigma}^{-}\right)_{L^{2}\left(\mathbb{R}^{d}\right)} \leq O\left(\sigma^{-2}\right)+O\left(\sigma^{-1}\right)\left\|k_{\sigma}^{-}\right\|_{H^{1}\left(\mathbb{R}^{d}\right)}$, and therefore

$$
\left\|k_{\sigma}^{-}\right\|_{H^{1}\left(\mathbb{R}^{d}\right)}=O(1 / \sigma) .
$$

Now, for any $z \in H^{1}$, we can estimate $J_{r, \mu}^{\prime \prime}\left(u_{n}\right) \cdot(z)^{2}$ from above:

$$
\begin{aligned}
J_{r, \mu}^{\prime \prime}\left(u_{n}\right) \cdot(z)^{2} \leq( & \left.\left.-\Delta+p(x)-\frac{\alpha}{|x|}\right) z, z\right)+\beta \iint_{\mathbb{R}^{d} \times \mathbb{R}^{d}} \frac{u_{n}^{2}(x) z(y)^{2}}{|x-y|} d x d y \\
& +2 \beta \iint_{\mathbb{R}^{d} \times \mathbb{R}^{d}} \frac{u_{n}(x) z(x) u_{n}(y) z(y)}{|x-y|} d x d y-\lambda_{n}\left\langle A_{\mu} z, z\right\rangle .
\end{aligned}
$$

The second member of this inequality is just $\left(z, L z-N^{\prime}\left(u_{n}\right) z-\lambda_{n} A_{\mu} z\right)_{H}$. If $z$ is fixed, then one easily proves that $\left(z, L z-N^{\prime}\left(u_{*}\right) z-\lambda_{*} A_{\mu} z\right)_{H}$ is the limit of $\left(z, L z-N^{\prime}\left(u_{n}\right) z-\lambda_{n} A_{\mu} z\right)_{H}$ as $n$ goes to infinity.

Some tedious but straightforward computations show that as $\sigma$ goes to $+\infty$,

$$
\int_{\mathbb{R}^{d}} \frac{\alpha}{|x|}\left|k_{\sigma}\right|^{2} d x=\frac{\alpha}{\sigma} M\left(\psi^{2}\right) \int_{\mathbb{R}^{d}} \frac{|f|^{2}}{|x|} d x+o\left(\frac{1}{\sigma}\right),
$$




$$
\begin{aligned}
\iint_{\mathbb{R}^{d} \times \mathbb{R}^{d}} \frac{u_{*}^{2}(x) k_{\sigma}^{2}(y)}{|x-y|} d x d y & =\int_{\mathbb{R}^{d}}\left\{\int_{\mathbb{R}^{d}} \frac{k_{\sigma}^{2}(y)}{|x-y|} d y\right\} u_{*}^{2}(x) d x \\
=\sigma^{-1} \int_{\mathbb{R}^{d}} & \left\{\int_{\mathbb{R}^{d}} \frac{f^{2}(y) \psi^{2}(\sigma y)}{|x / \sigma-y|} d y\right\} u_{*}^{2}(x) d x \\
& \leq\left\{\frac{1}{\sigma} M\left(\psi^{2}\right) \int_{\mathbb{R}^{d}} \frac{|f|^{2}}{|x|} d x+o\left(\frac{1}{\sigma}\right)\right\}\left\|u_{*}\right\|_{L^{2}\left(\mathbb{R}^{d}\right)}^{2},
\end{aligned}
$$

and again by (5.37),

$$
\left((-\Delta+p(x)-l) k_{\sigma}^{+}, k_{\sigma}^{+}\right)_{L^{2}\left(\mathbb{R}^{d}\right)} \leq\left((-\Delta+p-l) k_{\sigma}, k_{\sigma}-2 k_{\sigma}^{-}\right)_{L^{2}\left(\mathbb{R}^{d}\right)}=O\left(\frac{1}{\sigma^{2}}\right) .
$$

Now, we want to compute $F_{r, \mu}^{\prime \prime}\left(u_{n}\right) \cdot\left(k_{\sigma}^{+}\right)^{2}$. By $(3.17)$,

$$
\left.F_{r, \mu}^{\prime \prime}\left(v_{n}\right) \cdot\left(k_{\sigma}^{+}\right)^{2}=J_{r, \mu}^{\prime \prime}\left(u_{n}\right) \cdot\left(k_{\sigma}^{+}+g_{r, \mu}^{\prime}\left(v_{n}\right) k_{\sigma}^{+}\right)\right)^{2} .
$$

In order to estimate from above the r.h.s. of the above equality, let us show that $\left\|g_{r, \mu}^{\prime}\left(v_{n}\right) k_{\sigma}^{+}\right\|_{H^{1}\left(\mathbb{R}^{d}\right)}$ is also small when $\sigma$ is large.

By (3.15), we have

$$
\begin{gathered}
L\left(g_{r, \mu}^{\prime}\left(v_{n}\right) k_{\sigma}^{+}\right)-(I-P) N^{\prime}\left(u_{n}\right)\left(k_{\sigma}^{+}+g_{r, \mu}^{\prime}\left(v_{n}\right) k_{\sigma}^{+}\right)-2 f_{r}^{\prime}\left(\left\langle A_{\mu} u_{n}, u_{n}\right\rangle\right) A_{\mu}\left(g_{r, \mu}^{\prime}\left(v_{n}\right) k_{\sigma}^{+}\right) \\
-4 f_{r}^{\prime \prime}\left(\left\langle A_{\mu} u_{n}, u_{n}\right\rangle\right)\left\langle A_{\mu} u_{n}, k_{\sigma}^{+}+g_{r, \mu}^{\prime}\left(v_{n}\right) k_{\sigma}^{+}\right\rangle A_{\mu} g_{r, \mu}\left(v_{n}\right)=0 .
\end{gathered}
$$

Assuming that $\left\langle A_{\mu} v_{n}, k_{\sigma}\right\rangle=0$, the above equality together with (1.5) and the properties of $f_{r}$ give

$$
\begin{gathered}
\text { Const }\left\|g_{r, \mu}^{\prime}\left(v_{n}\right) k_{\sigma}^{+}\right\|_{H^{1}\left(\mathbb{R}^{d}\right)}^{2} \\
\leq-\left\langle L\left(g_{r, \mu}^{\prime}\left(v_{n}\right) k_{\sigma}^{+}\right), g_{r, \mu}^{\prime}\left(v_{n}\right) k_{\sigma}^{+}\right\rangle+\left\langle N^{\prime}\left(u_{n}\right) g_{r, \mu}^{\prime}\left(v_{n}\right) k_{\sigma}^{+}, g_{r, \mu}^{\prime}\left(v_{n}\right) k_{\sigma}^{+}\right\rangle \\
=-\left\langle N^{\prime}\left(u_{n}\right) k_{\sigma}^{+}, g_{r, \mu}^{\prime}\left(v_{n}\right) k_{\sigma}^{+}\right\rangle-2 f_{r}^{\prime}\left(\left\langle A_{\mu} u_{n}, u_{n}\right\rangle\right)\left\langle A_{\mu}\left(g_{r, \mu}^{\prime}\left(v_{n}\right) k_{\sigma}^{+}\right), g_{r, \mu}^{\prime}\left(v_{n}\right) k_{\sigma}^{+}\right\rangle \\
-4 f_{r}^{\prime \prime}\left(\left\langle A_{\mu} u_{n}, u_{n}\right\rangle\right)\left\langle A_{\mu} u_{n}, k_{\sigma}^{+}+g_{r, \mu}^{\prime}\left(v_{n}\right) k_{\sigma}^{+}\right\rangle\left\langle A_{\mu} g_{r, \mu}\left(v_{n}\right), g_{r, \mu}^{\prime}\left(v_{n}\right) k_{\sigma}^{+}\right\rangle \\
\leq-\left\langle N^{\prime}\left(u_{n}\right) k_{\sigma}^{+}, g_{r, \mu}^{\prime}\left(v_{n}\right) k_{\sigma}^{+}\right\rangle .
\end{gathered}
$$

Now, using the fact that since by $(5.38),\left\|k_{\sigma}-k_{\sigma}^{+}\right\|_{H^{1}\left(\mathbb{R}^{d}\right)}=O\left(\sigma^{-1}\right)$ we easily see that

$$
\begin{aligned}
\left|\left\langle N^{\prime}\left(u_{n}\right) k_{\sigma}^{+}, g_{r, \mu}^{\prime}\left(v_{n}\right) k_{\sigma}^{+}\right\rangle\right| & \\
\leq & O\left(\sigma^{-1}\right)\left\|g_{r, \mu}^{\prime}\left(v_{n}\right) k_{\sigma}^{+}\right\|_{L^{2}\left(\mathbb{R}^{d}\right)}+\left|\left\langle N^{\prime}\left(u_{n}\right)\left|k_{\sigma}\right|, \mid g_{r, \mu}^{\prime}\left(v_{n}\right) k_{\sigma}^{+}\right\rangle\right| \\
\leq & \left(O\left(\sigma^{-1}\right)+\frac{\text { Const }}{\sigma}\|\psi\|_{L^{\infty}\left(\mathbb{R}^{d}\right)}\|\nabla f\|_{L^{2}\left(\mathbb{R}^{d}\right)}\right)\left\|g_{r, \mu}^{\prime}\left(v_{n}\right) k_{\sigma}^{+}\right\|_{L^{2}\left(\mathbb{R}^{d}\right)} \\
& =O\left(\sigma^{-1}\right)\left\|g_{r, \mu}^{\prime}\left(v_{n}\right) k_{\sigma}^{+}\right\|_{L^{2}\left(\mathbb{R}^{d}\right)} .
\end{aligned}
$$

Hence, putting together the two above inequalities, we get

$$
\left\|g_{r, \mu}^{\prime}\left(v_{n}\right) k_{\sigma}^{+}\right\|_{H^{1}\left(\mathbb{R}^{d}\right)}=O\left(\sigma^{-1}\right) .
$$


Finally, in order to estimate $\left.J_{r, \mu}^{\prime \prime}\left(u_{n}\right) \cdot\left(k_{\sigma}^{+}+g_{r, \mu}^{\prime}\left(v_{n}\right) k_{\sigma}^{+}\right)\right)^{2}$, we use Hardy inequality, (5.38) and (5.41) to obtain the following

$$
\begin{aligned}
& \int_{\mathbb{R}^{d}} \frac{\alpha}{|x|}\left|k_{\sigma}^{+}+g_{r, \mu}^{\prime}\left(v_{n}\right) k_{\sigma}^{+}\right|^{2} d x \int_{\mathbb{R}^{d}} \frac{\alpha}{|x|}\left|k_{\sigma}-k_{\sigma}^{-}+g_{r, \mu}^{\prime}\left(v_{n}\right) k_{\sigma}^{+}\right|^{2} d x \\
& =\int_{\mathbb{R}^{d}} \frac{\alpha}{|x|}\left|k_{\sigma}\right|^{2} d x-2 \int_{\mathbb{R}^{d}} \frac{\alpha}{|x|} k_{\sigma}\left(k_{\sigma}^{-}-g_{r, \mu}^{\prime}\left(v_{n}\right) k_{\sigma}^{+}\right) d x+\int_{\mathbb{R}^{d}} \frac{\alpha}{|x|}\left|k_{\sigma}^{-}-g_{r, \mu}^{\prime}\left(v_{n}\right) k_{\sigma}^{+}\right|^{2} d x \\
& =\int_{\mathbb{R}^{d}} \frac{\alpha}{|x|}\left|k_{\sigma}\right|^{2} d x+2 O\left(\sqrt{\int_{\mathbb{R}^{d}} \frac{\alpha}{|x|}\left|k_{\sigma}\right|^{2} d x \int_{\mathbb{R}^{d}} \frac{2 \alpha}{|x|}\left(\left|k_{\sigma}^{-}\right|^{2}+\left|g_{r, \mu}^{\prime}\left(v_{n}\right) k_{\sigma}^{+}\right|^{2}\right) d x}\right) \\
& +\int_{\mathbb{R}^{d}} \frac{2 \alpha}{|x|}\left(\left|k_{\sigma}^{-}\right|^{2}+\left|g_{r, \mu}^{\prime}\left(v_{n}\right) k_{\sigma}^{+}\right|^{2}\right) d x=\frac{\alpha}{\sigma} M\left(\psi^{2}\right) \int_{\mathbb{R}^{d}} \frac{|f|^{2}}{|x|} d x+o\left(\frac{1}{\sigma}\right), \\
& \iint_{\mathbb{R}^{d} \times \mathbb{R}^{d}} \frac{u_{n}^{2}(x) k_{\sigma}^{+}(y)^{2}}{|x-y|} d x d y \\
& \leq\left\{\frac{1}{\sigma} M\left(\psi^{2}\right) \int_{\mathbb{R}^{d}} \frac{|f|^{2}}{|x|} d x+o\left(\frac{1}{\sigma}\right)\right\}\left\|u_{*}\right\|_{L^{2}}^{2}+o(1)_{n \rightarrow \infty} \\
& \iint_{\mathbb{R}^{d} \times \mathbb{R}^{d}} \frac{u_{n}(x) k_{\sigma}^{+}(x) u_{n}(y) k_{\sigma}^{+}(y)}{|x-y|} d x d y \\
& =\iint_{\mathbb{R}^{d} \times \mathbb{R}^{d}} \frac{u_{*}(x) k_{\sigma}^{+}(x) u_{*}(y) k_{\sigma}^{+}(y)}{|x-y|} d x+o(1)_{n \rightarrow \infty}, \\
& \iint_{\mathbb{R}^{d} \times \mathbb{R}^{d}} \frac{u_{n}^{2}(x) k_{\sigma}^{-}(y)^{2}}{|x-y|} d x d y=O\left(\sigma^{-2}\right),
\end{aligned}
$$

and

$$
\iint_{\mathbb{R}^{d} \times \mathbb{R}^{d}} \frac{u_{n}^{2}(x)\left(g_{r, \mu}^{\prime}(v) k_{\sigma}^{+}\right)(y)^{2}}{|x-y|} d x d y=O\left(\sigma^{-2}\right) .
$$

Moreover we may split the integral

$$
\iint_{\mathbb{R}^{d} \times \mathbb{R}^{d}} \frac{u_{*}(x) k_{\sigma}(x) u_{*}(y) k_{\sigma}(y)}{|x-y|} d x d y
$$

as a sum of two integrals

$$
\begin{aligned}
I_{1} & =\iint_{\mathbb{R}^{d} \times \mathbb{R}^{d}} \frac{u_{*}(x) k_{\sigma}(x) 1_{|x| \leq \sqrt{\sigma}} u_{*}(y) k_{\sigma}(y)}{|x-y|} d x d y \\
& \leq\left\{\iint_{\mathbb{R}^{d} \times \mathbb{R}^{d}} \frac{k_{\sigma}^{2}(x) 1_{|x| \leq \sqrt{\sigma}} u_{*}^{2}(y)}{|x-y|} d x d y \iint_{\mathbb{R}^{d} \times \mathbb{R}^{d}} \frac{k_{\sigma}^{2}(y) u_{*}^{2}(x)}{|x-y|} d x d y\right\}^{1 / 2} \\
& \leq \frac{\text { Const }}{\sigma}\left\{\|\nabla f\|_{L^{2}\left(\mathbb{R}^{d}\right)}^{2}\left\|k_{\sigma} 1_{|x| \leq \sqrt{\sigma}}\right\|_{L^{2}\left(\mathbb{R}^{d}\right)}\left\|k_{\sigma}\right\|_{L^{2}\left(\mathbb{R}^{d}\right)}\right\}^{1 / 2}\left\|u_{*}\right\|_{L^{2}\left(\mathbb{R}^{d}\right)}^{2}
\end{aligned}
$$


and

$$
\begin{aligned}
I_{2} & =\iint_{\mathbb{R}^{d} \times \mathbb{R}^{d}} \frac{u_{*}(x) k_{\sigma}(x) 1_{|x|>\sqrt{\sigma}} u_{*}(y) k_{\sigma}(y)}{|x-y|} d x d y \\
& \leq\left\{\iint_{\mathbb{R}^{d} \times \mathbb{R}^{d}} \frac{k_{\sigma}^{2}(x) u_{*}^{2}(y)}{|x-y|} d x d y \iint_{\mathbb{R}^{d} \times \mathbb{R}^{d}} \frac{k_{\sigma}^{2}(y) 1_{|x|>\sqrt{\sigma}} u_{*}^{2}(x)}{|x-y|} d x d y\right\}^{1 / 2} \\
& \leq \text { Const } \sigma^{-1}\|\nabla f\|_{L^{2}\left(\mathbb{R}^{d}\right)}\left\|k_{\sigma}\right\|_{L^{2}\left(\mathbb{R}^{d}\right)}\left\{\int_{\mathbb{R}^{d}} u_{*}^{2}(y) d y \int_{\mathbb{R}^{d}} 1_{|x|>\sqrt{\sigma}} u_{*}^{2}(x) d x\right\}^{1 / 2}
\end{aligned}
$$

it is easy to see that each of these integrals is $o\left(\sigma^{-1}\right)$. As a consequence,

$$
\iint_{\mathbb{R}^{d} \times \mathbb{R}^{d}} \frac{u_{n}(x) k_{\sigma}^{+}(x) u_{n}(y) k_{\sigma}^{+}(y)}{|x-y|} d x d y=o\left(\sigma^{-1}\right)+o(1)_{n \rightarrow \infty} .
$$

The above estimates show that, for large enough $\sigma$,

$$
\begin{aligned}
& \limsup _{n \rightarrow+\infty} J_{r, \mu}^{\prime \prime}\left(u_{n}\right) \cdot\left(k_{\sigma}^{+}+g_{r, \mu}^{\prime}\left(v_{n}\right) k_{\sigma}^{+}\right)^{2} \\
& \leq l\left\|k_{\sigma}^{+}\right\|_{L^{2}\left(\mathbb{R}^{d}\right)}^{2}-\frac{\left(\alpha-\beta \int_{\mathbb{R}^{d}}\left|u_{*}\right|^{2} d x\right)}{\sigma} M\left(\psi^{2}\right) \int_{\mathbb{R}^{d}} \frac{|f|^{2}}{|x|} d x-\lambda_{*}\left\langle A_{\mu} k_{\sigma}^{+}, k_{\sigma}^{+}\right\rangle+o\left(\frac{1}{\sigma}\right) \\
& \quad \leq l-\frac{\left(\alpha-\beta \int_{\mathbb{R}^{d}}\left|u_{*}\right|^{2} d x\right)}{\sigma} M\left(\psi^{2}\right) \int_{\mathbb{R}^{d}} \frac{|f|^{2}}{|x|} d x+o\left(\frac{1}{\sigma}\right)-\lambda_{*}\left(1+O\left(\sigma^{-2}\right)\right) .
\end{aligned}
$$

The r.h.s. of the above inequality is negative if $\sigma$ is large enough and

$$
\lambda_{*}>l-\frac{\bar{C}(\alpha, \beta, \psi, f)}{\sigma},
$$

with $\bar{C}(\alpha, \beta, \psi, f)>0$.

We finally extend this method to a 3 -dimensional space $X$ by considering additional functions $\tilde{f}, \bar{f}$ such that $f, \tilde{f}, \bar{f}$ generate a 3 -dimensional space. Choosing $\sigma$ large enough, we find a constant $l^{\prime}<l$, which depends only on $\bar{\sigma}, \alpha, \beta, f, \tilde{f}, \bar{f}$ and $\psi$, such that the inequality $\lambda_{*}>l^{\prime}$ would imply that the negative space of $F_{r, \mu}^{\prime \prime}\left(v_{n}\right)+\delta_{n}$ has dimension at least 2 for $\mu$ small and $r$ and $n$ large, which contradicts our assumptions (the dimension being reduced from 3 to 2 because we assumed $\left.<A_{\mu} v_{n}, k_{\sigma}>=0\right)$. So we have proved (3.20) by contradiction.

Note that this kind of argument, relating the Morse index and the location of a nonlinear eigenvalue in a Hartree-Fock equation, was first used by P.L. Lions [15].

Second Step : Compactness holds for critical sequences satisfying (3.20) or (3.21).

Let $\left(r_{n}, \mu_{n}\right) \rightarrow(r, \mu) \in(2, \infty] \times[0,1]$ and let $\left(v_{n}\right)$ be a sequence satisfying $F_{r_{n}, \mu_{n}}^{\prime}\left(v_{n}\right) \rightarrow 0$ and

$$
\limsup _{n \rightarrow \infty} f_{r_{n}}^{\prime}\left(\left\langle A_{\mu_{n}} u_{n}, u_{n}\right\rangle\right)<l / 2
$$


with $u_{n}=v_{n}+g_{r_{n}, \mu_{n}}\left(v_{n}\right)$. Define also $w_{n}=g_{r_{n}, \mu_{n}}\left(v_{n}\right)$ and $\lambda_{n}=2 f_{r_{n}}^{\prime}\left(\left\langle A_{\mu_{n}} u_{n}, u_{n}\right\rangle\right)$. Thus, $\left(u_{n}\right) \subset H=H^{1}\left(\mathbb{R}^{d}\right)$ is a sequence such that $\int_{\mathbb{R}^{d}}\left|u_{n}\right|^{2} d x \leq 1$, and

$$
\left(-\Delta+p(x)-\frac{\alpha}{|x|}+\beta\left(u_{n}^{2} * \frac{1}{|x|}\right)\right) u_{n}=\lambda_{n} u_{n}+\varepsilon_{n}, \quad\left\|\varepsilon_{n}\right\|_{H^{-1}\left(\mathbb{R}^{d}\right)} \rightarrow_{n} 0 .
$$

To see that $\left(u_{n}\right)$ is bounded in $H$, multiply (5.43) by $u_{n}$ and integrate, to get

$$
\begin{aligned}
& \int_{\mathbb{R}^{d}}\left|\nabla u_{n}\right|^{2} d x \\
\leq & \left(\|p\|_{L^{\infty}\left(\mathbb{R}^{d}\right)}+\lambda_{n}\right) \int_{\mathbb{R}^{d}} u_{n}^{2} d x+\alpha \int_{\mathbb{R}^{d}}|x|^{-1} u_{n}^{2} d x+\left\|\varepsilon_{n}\right\|_{H^{-1}\left(\mathbb{R}^{d}\right)}\left\|u_{n}\right\|_{H^{1}\left(\mathbb{R}^{d}\right)} \\
\leq & \text { Const }\left\|u_{n}\right\|_{L^{2}\left(\mathbb{R}^{d}\right)}^{2}+\text { Const }\|u\|_{H^{1}\left(\mathbb{R}^{d}\right)}\left\|u_{n}\right\|_{L^{2}\left(\mathbb{R}^{d}\right)}+\left\|\varepsilon_{n}\right\|_{H^{-1}\left(\mathbb{R}^{d}\right)}\left\|u_{n}\right\|_{H^{1}\left(\mathbb{R}^{d}\right)} .
\end{aligned}
$$

As $\left(u_{n}\right)$ is bounded in $L^{2}\left(\mathbb{R}^{d}\right)$, it is therefore also bounded in $H^{1}\left(\mathbb{R}^{d}\right)$.

It is important to note that the operator $u \rightarrow|x|^{-1} u$ is compact from $H^{1}\left(\mathbb{R}^{d}\right)$ to its dual $H^{-1}\left(\mathbb{R}^{d}\right)$. Moreover $u \rightarrow\left(u^{2} *|x|^{-1}\right) u$ sends weakly convergent sequences in $H^{1}\left(\mathbb{R}^{d}\right)$ to weakly convergent sequences in $H^{-1}\left(\mathbb{R}^{d}\right)$ with corresponding limits (this is a consequence of the Hardy inequality and the presence of $|x|^{-1}$ ).

Extracting a subsequence if needed, we can assume that $u_{n} \rightarrow \bar{u}$ weakly in $H^{1}\left(\mathbb{R}^{d}\right)$ for some $\bar{u} \in H^{1}\left(\mathbb{R}^{d}\right)$ and $\lambda_{n} \rightarrow \bar{\lambda}$ for some $\bar{\lambda} \in[0, l)$. By the previous remarks, we get

$$
\left(-\Delta+p(x)-\frac{\alpha}{|x|}+\beta\left(\bar{u}^{2} * \frac{1}{|x|}\right)\right) \bar{u}=\bar{\lambda} \bar{u}, \quad \int_{\mathbb{R}^{d}} \bar{u}^{2} d x \leq 1 .
$$

Hence, setting $\bar{u}_{n}=u_{n}-\bar{u}$,

$$
\begin{aligned}
\left(-\Delta+p(x)-\frac{\alpha}{|x|}-\lambda_{n}\right) u_{n}-(-\Delta & \left.+p(x)-\frac{\alpha}{|x|}-\bar{\lambda}\right) \bar{u} \\
& =\varepsilon_{n}-\beta\left(u_{n}^{2} * \frac{1}{|x|}\right) u_{n}+\beta\left(\bar{u}^{2} * \frac{1}{|x|}\right) \bar{u}
\end{aligned}
$$

and

$$
\begin{gathered}
\left(-\Delta+p(x)-\frac{\alpha}{|x|}+\beta\left(\bar{u}_{n}^{2} *|x|^{-1}\right)-\bar{\lambda}\right) \bar{u}_{n}=\left(\lambda_{n}-\bar{\lambda}\right) u_{n}+\varepsilon_{n} \\
-\beta\left(\bar{u}^{2} * \frac{1}{|x|}\right) \bar{u}_{n}-\beta\left(2\left(\bar{u}_{n} \bar{u}\right) * \frac{1}{|x|}\right) \bar{u}_{n}-\beta\left(\bar{u}_{n}^{2} * \frac{1}{|x|}\right) \bar{u}-\beta\left(2\left(\bar{u}_{n} \bar{u}\right) * \frac{1}{|x|}\right) \bar{u} .
\end{gathered}
$$

Note that the right-hand member converges to 0 strongly in $H^{-1}\left(\mathbb{R}^{d}\right)$, so that

$$
\left(-\Delta+p(x)-\frac{\alpha}{|x|}+\beta\left(\bar{u}_{n}^{2} *|x|^{-1}\right)-\bar{\lambda}\right) \bar{u}_{n} \rightarrow 0
$$

strongly in $H^{-1}\left(\mathbb{R}^{d}\right)$. 
Define the operator $L_{n}: H^{1}\left(\mathbb{R}^{d}\right) \rightarrow H^{-1}\left(\mathbb{R}^{d}\right)$ by

$$
L_{n}=-\Delta+p(x)-\frac{\alpha}{|x|}+\beta\left(\bar{u}_{n}^{2} *|x|^{-1}\right)-\bar{\lambda} .
$$

Equation (5.44) can be written $L_{n} \bar{u}_{n} \rightarrow 0$ (strongly in $H^{-1}\left(\mathbb{R}^{d}\right)$ ).

Now, by (1.5), the map

$$
w \in H_{-} \mapsto G_{n}(w):=\left(\bar{u}_{n}+w, L_{n}\left(\bar{u}_{n}+w\right)\right)_{L^{2}}
$$

is strictly concave. So, denoting $\bar{v}_{n}=P \bar{u}_{n}, \bar{w}_{n}=(I-P) \bar{u}_{n}$,

$$
G_{n}\left(-\bar{w}_{n}\right) \leq G_{n}(0)-G_{n}^{\prime}(0) \cdot \bar{w}_{n} \leq 3\left\|\bar{u}_{n}\right\|_{H^{1}\left(\mathbb{R}^{d}\right)}\left\|L_{n} \bar{u}_{n}\right\|_{H^{-1}\left(\mathbb{R}^{d}\right)} .
$$

Hence, $\limsup _{n} G_{n}\left(-\bar{w}_{n}\right) \leq 0$. As $G_{n}\left(-\bar{w}_{n}\right)=\left(\bar{v}_{n}, L_{n} \bar{v}_{n}\right)_{L^{2}}$ and $|x|^{-1} \bar{v}_{n}$ converges strongly to 0 in $H^{-1}\left(\mathbb{R}^{d}\right)$, we have

$$
\underset{n}{\limsup }\left(\bar{v}_{n},\left(L_{n}+\alpha|x|^{-1}\right) \bar{v}_{n}\right)_{L^{2}} \rightarrow 0 .
$$

As, by (1.5),

$$
\left(\bar{v}_{n},\left(L_{n}+\alpha|x|^{-1}\right) \bar{v}_{n}\right)_{L^{2}} \geq h_{0}\left\|\bar{v}_{n}\right\|_{H^{1}\left(\mathbb{R}^{d}\right)}^{2}
$$

for some $h_{0}>0$, we deduce $\bar{v}_{n} \rightarrow 0$ strongly in $H^{1}\left(\mathbb{R}^{d}\right)$ and $\left(\bar{w}_{n}, L_{n} \bar{w}_{n}\right)_{L^{2}} \rightarrow 0$.

But again by (1.5),

$$
\left(\bar{w}_{n}, L_{n} \bar{w}_{n}\right)_{L^{2}} \leq-h_{0}\left\|\bar{w}_{n}\right\|_{H^{1}\left(\mathbb{R}^{d}\right)}^{2}
$$

for some $h_{0}>0$. Hence, $\left\|\bar{w}_{n}\right\|_{H^{1}\left(\mathbb{R}^{d}\right)} \rightarrow 0$ and

$$
\left\|\bar{u}_{n}\right\|_{H^{1}\left(\mathbb{R}^{d}\right)}=\left\|u_{n}-\bar{u}\right\|_{H^{1}\left(\mathbb{R}^{d}\right)} \underset{n \rightarrow+\infty}{\longrightarrow} 0 .
$$

\section{Last Step.}

Let us now check that the second case of the alternative in Theorem 5 does not occur and that, in Theorem $4, \lambda>\lambda_{0}$ with $\lambda_{0}=0$ (without loss of generality). Define the operator $\tilde{L}_{u}: H^{1}\left(\mathbb{R}^{d}\right) \rightarrow H^{-1}\left(\mathbb{R}^{d}\right)$ by

$$
\tilde{L}_{u}=-\Delta+p(x)-\frac{\alpha}{|x|}+\beta\left(u^{2} *|x|^{-1}\right)
$$

for $u \in H^{1}\left(\mathbb{R}^{d}\right)$ such that $0<\int_{\mathbb{R}^{d}} u^{2} d x \leq 1$, assume that $\tilde{L}_{u} u=0$ and let us look for a contradiction. By (1.5), the map

$$
w \in H_{-} \mapsto G_{u}(w):=\left(u+w, \tilde{L}_{u}(u+w)\right)_{L^{2}}
$$

is strictly concave. Letting $v=P u, w=(I-P) u$, we get

$$
G_{u}(-w) \leq G_{u}(0)-G_{u}^{\prime}(0) \cdot w \leq 3\|u\|_{H^{1}\left(\mathbb{R}^{d}\right)}\left\|\tilde{L}_{u} u\right\|_{H^{-1}\left(\mathbb{R}^{d}\right)}=0
$$

and $G_{u}(-w) \leq 0$. As $G_{u}(-w)=\left(v, \tilde{L}_{u} v\right)_{L^{2}}$, we get $v=0$, thanks to (1.5). Hence $\left(w, \tilde{L}_{u} w\right)_{L^{2}}=0$ and $w=0$ (by (1.5) again), which is in contradiction with $u \neq 0$. 


\section{References}

[1] H. Amann, Saddle points and multiple solutions of differential equations, Math. Z. 169 (1979), 127-166.

[2] A.S. Besicovich, Almost Periodic Functions, Cambridge Univ. Press, Cambridge, 1932.

[3] B. Buffoni and L. Jeanjean, Minimax characterization of solutions for a semi-linear elliptic equation with lack of compactness, Ann. Inst. Henri Poincaré 10 (1993), 377404.

[4] B. Buffoni, L. Jeanjean and C. A. Stuart, Existence of a nontrivial solution to a strongly indefinite semilinear equation, Proceedings of the AMS 119 (1993), 179-186.

[5] A. Castro, Méthodes de réduction via minimax, in Differential Equations, Lect. Notes Math. no 957, Springer, Berlin, 1982.

[6] A. Castro and A. C. Lazer, Applications of a min-max principle, Rev. Colomb. Mat. 10 (1976), 141-149.

[7] M. J. Esteban and E. Séré, Solutions of the Dirac-Fock equations for atoms and molecules, Comm. Math. Phys. 203 (1999), 499-530.

[8] R. P. Feynman, The Feynman Lectures on Physics 3 : Quantum Mechanics. AddisonWesley, 1965.

[9] N. Ghoussoub, Duality and Perturbation Methods in Critical Point Theory, Cambridge University Press, 1993.

[10] H. P. Heinz, Lacunary bifurcation for operator equations and nonlinear boundary value problems on $\mathbb{R}^{d}$, Proc. Royal Soc. Edinburgh 118 (1991), 237-270.

[11] H. P. Heinz, T. Küpper and C. A. Stuart, Existence and bifurcation of solutions for nonlinear perturbations of the periodic Schrödinger equation, J. Diff. Eqns 100 (1992), 341-354.

[12] H. P. Heinz and C. A. Stuart, Solvability of Nonlinear Equation in Spectral Gaps of the Linearisation, Nonlinear Analysis - TMA 19 (1992), 145-165.

[13] D. B. Hinton and J. K. Shaw, On the absolutely continuous spectrum of the pertubed Hill's equation, Proc. Lond. Math. Soc. (Third Series) 50 (1985), 175-192.

[14] T. Küpper and C. A. Stuart, Gap-bifurcation for nonlinear perturbations of Hill's equation, J. reine angew. Math. 410 (1990), 23-52.

[15] P.-L. Lions. Solutions of Hartree-Fock equations for Coulomb systems. Comm. Math. Phys. 109 (1987), p. 33-97.

[16] F. S. Rofe-Beketov, A test for the finiteness of the number of discrete levels introduced into the gaps of a continuous spectrum by perturbations of a periodic potential, Soviet. Math. Dokl. 5 (1964), 689-692.

[17] C.A. Stuart, Bifurcation Into Spectral Gaps, Société Mathématique de Belgique, 1995. 